\title{
Arithmetic of alternating forms and quaternion hermitian forms
}

\author{
By Goro SHIMURA
}

(Received June 21, 1962)

Hecke's Dirichlet series obtained from modular forms can be regarded as zeta-functions attached to the general linear group $G L(2, \boldsymbol{Q})$ over the rational number field $\boldsymbol{Q}$. In general, we may expect to obtain zeta-functions of this kind for a fairly wide class of algebraic groups defined over $\boldsymbol{Q}$. In order to realize this, it is necessary to develop, in the first place, the theory of elementary divisors for any algebraic group $G$ in question. This is actually done in the case where $G$ is the multiplicative group of a semi-simple algebra. Further, the case of the orthogonal group is investigated in detail by M. Eichler [3]. In both cases, there are fundamental theorems, due to Eichler [4, 5] and M. Kneser [6], which may be called the approximation theorem in the group. $G$, from which one can easily derive an important conclusion about the classnumber for $G$. This approximation theorem plays an essential role also in the theory of Hecke-rings attached to quaternion algebras [8, 9]. In fact, by means of the theorem, we can prove the isomorphism between the Hecke-ring defined by the idele-group of a quaternion algebra $D$ and the Hecke-ring: defined by the unit-groups of maximal orders in $D$ (cf. $[9, \S 2]$ ).

The purpose of the present paper is to give an extension of the theory of elementary divisors for the group of similitudes of a hermitian form over a quaternion algebra, and to prove an approximation theorem for this group. Let $F$ be the quotient field of a Dedekind domain $g$ and $A$ a quaternion (not necessarily division) algebra over $F$. Let $V$ be a left $A$-module which is isomorphic to the product of $n$ copies of $A$. We consider an $A$-valued non-degenerate hermitian form $f(x, y)$ on $V$ with respect to the canonical involution. of $A$ (cf. $\S 2.2$ ). Let $G$ be the group consisting of all $A$-automorphisms $\sigma$ of $V$ such that $f(x \sigma, y \sigma)=N(\sigma) f(x, y)$ for $x \in V, y \in V$ with $N(\sigma) \in F$. Take a maximal oder $\mathfrak{D}$ in $A$. Let $L$ be a $g$-lattice in $V$ such that $\mathfrak{o} \subset \subset L$. We denote: by $N(L)$ the two-sided $\mathfrak{D}$-ideal generated by $f(x, y)$ for $x \in L, y \in L$, and call it the norm of $L$. We say that $L$ is maximal if $L$ is a maximal one among the lattices with the same norm. As in [3], our theory is mostly concerned with maximal lattices in $V$. If $A$ is the total matric algebra of degree 2 over $F$, then $G$ is isomorphic to the group of similitudes of an alternating: 
form over $F$ with $2 n$ variables. We treat this case in $\S 1$ and $\S 2.5$, and prove fundamental propositions concerning the existence of canonical bases for maximal lattices and their elementary divisors. We give in $\S 3$ similar propositions in case where $A$ is a division quaternion algebra over a $\mathfrak{p}$-adic field. These propositions correspond to the results of the same kind obtained in the case of orthogonal groups [Eicher, 3$]$ and of quaternion anti-hermitian forms [Tsukamoto, 10]. In $\S 4$, we consider the global theory, namely the case where $F$ is an algebraic number field. Our principal aim is to prove approximationtheorems for $G$ (Theorems 1 and 2 of $\S 4.6$ ) in case where $A$ is indefinite. As an application of the theorems, we can show that the classes of maximal lattices in each genus are in one-to-one correspondence with the ideal-classes modulo $t$ in $F$ for a suitable product $t$ of infinite prime spots of $F$ (Theorem 3 ). If we denote by $G^{0}$ the unitary group of $f$, i. e. the subgroup of $G$ composed of the elements $\sigma$ such that $N(\sigma)=1$, then each genus with respect to $G^{0}$ consists of only one class ( $\left.\$ 4.9\right)$. Finally we give a result on global set of elementary divisors of maximal lattices (Theorem 4). As explained in the beginning, our theory can be considered as preliminaries for the theory of the Hecke-ring of $G$. In fact, by means of our propositions and theorems, we can develop such a theory, which is a generalization of the theory in $[\mathbf{9}, \S 2]$. As for this, we have only given Proposition 4.11. A further investigation of the Hecke-ring of $G$ will be made in a subsequent paper.

Notation. We denote by $\boldsymbol{Z}, \boldsymbol{Q}, \boldsymbol{R}, \boldsymbol{C}$ and $\boldsymbol{K}$, respectively, the ring of rational integers, the rational number field, the real number field, the complex number field, and the division ring of real quaternions. For a ring $S$ with an identity element, $M_{m}(S)$ denotes the ring of matrices of degree $m$ with entries in $S$; the identity matrix of degree $m$ is denoted by $1_{m}$; and the transpose of a matrix $X$ is denoted by ${ }^{t} X$. We mean by $\delta_{i j}$ the usual Kronecker's delta, namely $\delta_{i j}=0$ or 1 according as $i \neq j$ or $i=j$.

\section{$\S 1$. Arithmetic of alternating forms.}

1.1. Alternating form and symplectic group. Let $F$ be an arbitrary field and $W$ a vector space over $F$ of finite dimension. We denote by $E(W)$ the ring of all $F$-linear mappings of $W$ into itself, and by $G L(W)$ the group of regular elements of $E(W)$. We write the operation of an element of $E(W)$ on the right; so we have $(a x) \sigma=a(x \sigma)$ for $a \in F, x \in W, \sigma \in E(W)$. Let $g(x, y)$ be a non-degenerate alternating form on $W$. We denote by $G(W, g)$ the subgroup of $G L(W)$ consisting of the elements $\sigma$ of $G L(W)$ for which there exists a number $N(\sigma)$ of $F$ such that $g(x \sigma, y \sigma)=N(\sigma) g(x, y)$ for every $x, y \in W$, and denote by $G^{0}(W, g)$ the symplectic group associated to $g$, namely, the subgroup of $G(W, g)$ consisting of the elements $\sigma$ such that $N(\sigma)=1$. 
1.2. Lattices in a vector space. Let $g$ be a Dedekind domain and $F$ the quotient field of g. Let $W$ be a vector space over $F$. By a g-lattice in $W$, we understand a finitely generated $g$-submodule $L$ of $W$ such that $F L=W$. Let $\mathfrak{p}$ be a prime ideal of $\mathfrak{g}$. We denote by $F_{\mathfrak{p}}$ and $\mathfrak{g}_{\mathfrak{p}}$ the $\mathfrak{p}$-completions of $F$ and $\mathfrak{g}$, respectively. Put $W_{p}=W \underset{F}{\bigotimes} F_{\mathfrak{p}}$. For every g-lattice $L$ in $W$, put $L_{p}=\mathfrak{g}_{p} L$; then $L_{\mathfrak{p}}$ is a $\mathfrak{g}_{p}$-lattice in $W_{\mathfrak{p}}$. The following lemma is well-known.

LemMA 1.1. Let $L$ be a $\mathfrak{g}$-lattice in $W$. Take, for each prime ideal $\mathfrak{p}$ of $\mathfrak{g}$, $a \mathfrak{g}_{\mathfrak{p}}$-lattice $M^{\mathfrak{p}}$ in $W_{\mathfrak{p}}$. Then there exists a $\mathrm{g}$-lattice $M$ in $W$ such that $M_{\mathfrak{p}}=M^{\mathfrak{p}}$ for every $\mathfrak{n}$, if and only if $M^{\mathfrak{p}}=L_{\mathfrak{p}}$ for all except a finite number of $\mathfrak{p}$. If such a lattice $M$ exists, we have $M=\bigcap_{p}\left(M_{p} \cap W\right)$.

Lemma 1.2. Let $L_{p}$ be a $g_{p}$-lattice in $W_{p}$; let $\sigma$ and $\tau$ be elements of $G L\left(W_{p}\right)$. Suppose that $L_{p}(\sigma-\tau) \subset p L_{p} \sigma$. Then we have $L_{p} \sigma=L_{p} \tau$.

PROOF. Let $x_{1}, \cdots, x_{m}$ be generators of $L_{p}$ over $\mathfrak{g}_{\mathfrak{p}}$. Put $M=L_{p} \sigma, K=L_{p} \tau$. Then we have $x_{i} \sigma-x_{i} \tau \in \mathfrak{p} M \subset M$ for every $i$. As $M$ and $K$ are respectively generated by the $x_{i} \sigma$ and the $x_{i} \tau$, we get $K \subset M$. Further we have $M \subset K+\mathfrak{p} M$. From this we obtain inductively $M \subset K+p^{e} M$ for every positive integer $e$. This implies $M \subset K$, so that $M=K$.

1.3. Canonical base of a lattice with respect to an alternating form. We first prove a generalization of a well-known theorem of Frobenius.

Proposition 1.3. Let $\mathrm{g}$ be a Dedekind domain and $F$ the quotient field of g. Let $W$ be a vector space of dimension $2 n$ over $F$ and $g(x, y)$ a non-degenerate alternating form on $W$. Let $M$ be a g-lattice in $W$. Then there exist a base $\left\{y_{1}, \cdots, y_{n}, z_{1}, \cdots, z_{n}\right\}$ of $W$ over $F$ and (fractional) g-ideals $\mathfrak{a}_{1}, \cdots, \mathfrak{a}_{n}$ such that

$$
\begin{gathered}
g\left(y_{i}, y_{j}\right)=g\left(z_{i}, z_{j}\right)=0, \quad g\left(y_{i}, z_{j}\right)=\delta_{i j}, \\
M=\mathfrak{g} y_{1}+\mathfrak{g} y_{2}+\cdots+\mathfrak{g} y_{n}+\mathfrak{a}_{1} z_{1}+\mathfrak{a}_{2} z_{2}+\cdots+\mathfrak{a}_{n} z_{n}, \\
\mathfrak{a}_{1} \supset \mathfrak{a}_{2} \supset \cdots \supset \mathfrak{a}_{n} .
\end{gathered}
$$

The ideals $\mathfrak{a}_{i}$ are uniquely determined by $M$ and $g$.

PROOF. We prove this by induction on $n$. For every $x \in M$, put $\mathfrak{a}_{x}=g(x, M)$. Obviously, $\mathfrak{a}_{x}$ is a $\mathfrak{g}$-ideal. As $M$ is a $\mathfrak{g}$-lattice, there exists a maximal one among the $\mathfrak{a}_{x}$, say $\mathfrak{a}_{1}$; and take an element $y_{1}$ of $M$ so that $\mathfrak{a}_{1}=g\left(y_{1}, M\right)$. As we have $g=g\left(y_{1}, \mathfrak{a}_{1}^{-1} M\right)$, there exists an element $z_{1}$ of $\mathfrak{a}_{1}^{-1} M$ such that $g\left(y_{1}, z_{1}\right)$ $=1$. Put $\mathfrak{b}=g\left(M, z_{1}\right)$. As $\mathfrak{b} \ni g\left(y_{1}, z_{1}\right)=1$, we have $\mathfrak{b} \supset \mathfrak{g}$, so that $\mathfrak{a}_{1} \mathfrak{b}=\mathfrak{a}_{1} g\left(M, z_{1}\right)$ $\supset \mathfrak{a}_{1}$. Assume that $\mathfrak{b} \neq \mathfrak{g}$. Then $\mathfrak{a}_{1} g\left(M, z_{1}\right) \neq \mathfrak{a}_{1}$, and hence there exist an element $u$ of $M$ and an element $\alpha$ of $a_{1}$ such that $g\left(u, \alpha z_{1}\right) \notin \mathfrak{a}_{1}$. Put $\beta$ $=-g\left(u, \alpha z_{1}\right), \gamma=g\left(y_{1}, u\right)$. We have then $g\left(y_{1}+\alpha z_{1}, u-\gamma z_{1}\right)=\beta$. Since $\gamma \in a_{1}$ and $a_{1} z_{1} \subset M$, the element $u-\gamma z_{1}$ is contained in $M$. We note that $g\left(y_{1}+\alpha z_{1}, a_{1} z_{1}\right)$ $=a_{1}$. Therefore, we have

$$
g\left(y_{1}+\alpha z_{1}, M\right) \supset \mathfrak{a}_{1}+\mathfrak{g} \beta \supset \mathfrak{a}_{1}, \quad \mathfrak{a}_{1}+\mathfrak{g} \beta \neq \mathfrak{a}_{1} .
$$


This contradicts the maximality of $a_{1}$. Hence we must have $g\left(M, z_{1}\right)=\mathrm{g}$. Now define a submodule $M^{\prime}$ of $M$ by $M^{\prime}=\left\{v \in M \mid g\left(y_{1}, v\right)=g\left(z_{1}, v\right)=0\right\}$. For every $w \in M$, put $\xi=g\left(y_{1}, w\right), \eta=g\left(z_{1}, w\right), w_{0}=w+\eta y_{1}-\xi z_{1}$. Then $\xi \in \mathfrak{a}_{1}, \eta \in \mathfrak{g}$, and we have $g\left(y_{1}, w_{0}\right)=g\left(z_{1}, w_{0}\right)=0$, so that $w_{0} \in M^{\prime}$. This shows that $M$ $=g y_{1}+a_{1} z_{1}+M^{\prime}$. Applying our induction to $M^{\prime}$, we get an expression $M^{\prime}$ $=\mathfrak{g} y_{2}+\cdots+\mathfrak{g} y_{n}+\mathfrak{a}_{2} z_{2}+\cdots+\mathfrak{a}_{n} z_{n}$ with the properties $\mathfrak{a}_{2} \supset \cdots \supset \mathfrak{a}_{n}, g\left(y_{i}, y_{j}\right)=g\left(z_{i}, z_{j}\right)$ $=0, g\left(y_{i}, z_{j}\right)=\delta_{i j}$ for $2 \leqq i \leqq n, 2 \leqq j \leqq n$. Therefore, the first assertion is proved if we show $\mathfrak{a}_{1} \supset \mathfrak{a}_{2}$. Let $u$ and $v$ be elements of $M^{\prime}$. We have $g\left(y_{1}+u, M\right)$ $\supset g\left(y_{1}+u, a_{1} z_{1}+g v\right) \supset \mathfrak{a}_{1}+g g(u, v) \supset \mathfrak{a}_{1}$. By the maximality of $\mathfrak{a}_{1}$, we must have $g(u, v) \in \mathfrak{a}_{1}$, namely $g\left(M^{\prime}, M^{\prime}\right) \subset \mathfrak{a}_{1}$. This implies $\mathfrak{a}_{1} \supset \mathfrak{a}_{2}$ and completes the proof of the first assertion. The invariance of the ideals $\mathfrak{a}_{i}$ is easily shown by “localization". Namely, for every prime ideal $\mathfrak{p}$ of $\mathfrak{g}$, consider $W_{\mathfrak{p}}=W \underset{F}{\otimes} F_{\mathrm{p}}$ and a $g_{p}$-lattice $M_{\mathfrak{p}}=\mathfrak{g}_{p} M$ in $W_{p}$. Then the invariance is an immediate consequence of the theory of elementary divisors over a principal ideal domain (cf. [2, §5.1, Theorem 1]). We can also prove the invariance more directly with no use of localization.

We call the ideals $\mathfrak{a}_{i}$ of Proposition 1.3 the invariant factors of $M$ (with respect to $g$ ), and call $\left\{y_{1}, \cdots, y_{n}, z_{1}, \cdots, z_{n}\right\}$ a canonical base of $M$ (with respect to $g$ ).

1.4. Maximal lattices. Let $F, \mathfrak{g}, W, g$ be the same as in Proposition 1.3. For every $g$-lattice $M$ in $W$, we see that the first member $\mathfrak{a}_{1}$ of the invariant factors of $M$ is the $g$-ideal generated by $g(x, y)$ for $x, y \in M$. We put $N_{g}(M)$ $=\mathfrak{a}_{1}$ and call $N_{g}(M)$ the norm of $M$ with respect to $g$. For simplicity, we fix $g$ and write $N(M)=N_{g}(M)$. We say that $M$ is maximal (with respect to $g$ ) if $M$ is a maximal one among the g-lattices in $W$ with the same norm (with respect to $g$ ). It is clear that $N(M \sigma)=N(M) N(\sigma)$ for every $\sigma \in G(W, g)$. If $M$ is maximal, $M \sigma$ is maximal for every $\sigma \in G(W, g)$. By Proposition 1.3, we. see easily that $M$ is maximal if and only if the invariant factors of $M$ are all equal to $N(M)$. Furthermore, if $M$ is a $\mathfrak{g}$-lattice in $W$ and $\mathfrak{a}$ is a g-ideal such that $\mathfrak{a} \supset N(M)$, we can find a maximal lattice $L$ in $W$ such that $L \supset M$, $N(L)=\mathfrak{a}$.

Proposition 1.4. Let $M_{1}$ and $M_{2}$ be maximal lattices in $W$. Then we have $M_{1} \sigma=M_{2}$ for an element $\sigma$ of $G(W, g)$, if and only if $N\left(M_{1}\right)^{-1} N\left(M_{2}\right)$ is a principal ideal.

Proof. If $M_{1} \sigma=M_{2}$ for an element $\sigma \in G(W, g)$, we have $N\left(M_{2}\right)=N\left(M_{1} \sigma\right)$, $=N\left(M_{1}\right) N(\sigma)$; this proves the 'only if' part. Conversely, put $\mathfrak{a}_{i}=N\left(M_{i}\right)$ and $\mathfrak{a}_{1}^{-1} \mathfrak{a}_{2}=g \alpha$ with $\alpha \in F$. Let $\left\{y_{1}, \cdots, y_{n}, z_{1}, \cdots, z_{n}\right\}$ and $\left\{u_{1}, \cdots, u_{n}, v_{1}, \cdots, v_{n}\right\}$ be respectively canonical bases of $M_{1}$ and $M_{2}$. Define an element $\sigma$ of $E(W)$ by $y_{i} \sigma=u_{i}, z_{i} \sigma=\alpha v_{i}$ for $1 \leqq i \leqq n$. Then we see easily $\sigma \in G(W, g), N(\sigma)=\alpha, M_{1} \sigma$ $=M_{2}$. This proves the 'if' part. 
We say that maximal lattices $M_{1}$ and $M_{2}$ in $W$ are equivalent if $M_{1}=M_{2} \sigma$ for an element $\sigma$ of $G(W, g)$, and call a maximal set of mutually equivalent maximal lattices a class of maximal lattices. By Proposition 1.4, we observe that the mapping $M \rightarrow N(M)$ gives a one-to-one correspondence between the classes of maximal lattices in $W$ and the ideal-classes of $F$.

1.5. Invariant factors of elements of $\boldsymbol{G}(\boldsymbol{W}, \boldsymbol{g})$. Notation being as in $\S \S 1.3$ -4 , suppose that $g$ is a principal ideal domain.

Proposition 1.5. Let $L$ and $M$ be maximal lattices in $W$. Let $\alpha$ be an element of $F$ such that $N(M)=\alpha N(L)$. Put $N(L)=a$. Then there exist a canonical base $\left\{y_{1}, \cdots, y_{n}, z_{1}, \cdots, z_{n}\right\}$ of $L$ and elements $a_{1}, \cdots, a_{n}, b_{1}, \cdots, b_{n}$ of $F$ such that

$$
\begin{aligned}
& L=\mathfrak{g} y_{1}+\cdots+\mathfrak{g} y_{n}+\mathfrak{a} z_{1}+\cdots+\mathfrak{a} z_{n}, \\
& M=\mathfrak{g} a_{1} y_{1}+\cdots+\mathfrak{g} a_{n} y_{n}+\mathfrak{a} b_{1} z_{1}+\cdots+\mathfrak{a} b_{n} z_{n}, \\
& \alpha=a_{1} b_{1}=\cdots=a_{n} b_{n}, \\
& \mathfrak{g} a_{1} \supset \cdots \supset \mathfrak{g} a_{n} \supset \mathfrak{g} b_{n} \supset \cdots \supset \mathfrak{g} b_{1} .
\end{aligned}
$$

Proof. We proceed by induction on $n$. Put $c=\{c \in F \mid c M \subset L\}$. It is easy to see that $c$ is a $g$-ideal. As $g$ is a principal ideal domain, we have $\mathrm{c}=\mathrm{g} c_{0}$ for an element $c_{0}$ of $F$. Put $M^{\prime}=c_{0} M$. Then $M^{\prime}$ is a maximal lattice, and $N\left(M^{\prime}\right)=c_{0}{ }^{2} \alpha \mathfrak{a}, \mathfrak{g}=\left\{c \in F \mid c M^{\prime} \subset L\right\}$. If we prove our proposition for $M^{\prime}$ and $c_{0}{ }^{2} \alpha$, we get easily the assertion for $M$ and $\alpha$. Therefore, we may assume that $M=M^{\prime}$, namely, $\mathfrak{g}=\{c \in F \mid c M \subset L\}$. The last relation implies that $L \supset M$ and $M$ contains an element $y_{1} \neq 0$ such that $L / g y_{1}$ is a free $g$-module. Put $M_{1}=M+\alpha L$. Then $M_{1}$ is a $g$-lattice in $W$. As $L \supset M$ and $N(M)=\alpha N(L)$, we must have $\alpha \in \mathfrak{g}$; hence we see easily $N\left(M_{1}\right)=\alpha N(L)=N(M)$. As $M$ is maximal, we must have $M=M_{1}$, so that $M \supset \alpha L$. Now taking a canonical base of $L$, and expressing $y_{1}$ in a linear form of the base, we find that $g\left(y_{1}, L\right)$ $=a$. By the proof of Proposition 1.3, we can find an element $z_{1}$ of $\mathfrak{a}^{-1} L$ such that $g\left(y_{1}, z_{1}\right)=1$; and if we put $U=\left\{x_{0} \in W \mid g\left(y_{1}, x\right)=g\left(z_{1}, x\right)=0\right\}, L_{0}=L \cap U$, we get $L=\mathfrak{g} y_{1}+\mathfrak{a} z_{1}+L_{0}$. We see easily that $L_{0}$ is a maximal lattice in $U$ and $N\left(L_{0}\right)=\mathfrak{a}$. As $\alpha L \subset M$, we have $\alpha a z_{1} \subset M$. For every $x \in M$, we have $g\left(y_{1}, x\right)$ $\in N(M)=\alpha \mathfrak{a}, g\left(z_{1}, x\right) \in \mathfrak{a}^{-1} N(L)=g$. Hence if we put $g\left(y_{1}, x\right)=\xi \alpha, g\left(z_{1}, x\right)=\eta$, then $\xi \in \mathfrak{a}, \eta \in \mathfrak{g}$. Put $x_{0}=x+\eta y_{1}-\xi \alpha z_{1}$. We have then $x_{0} \in M$ and $g\left(y_{1}, x_{0}\right)$ $=g\left(z_{1}, x_{0}\right)=0$, so that $x_{0} \in U \cap M$. This proves that $M=\mathfrak{g} y_{1}+a \alpha z_{1}+M_{0}$, if we put $M_{0}=U \cap M$. As $M$ is maximal, $M_{0}$ must be a maximal lattice in $U$ such that $N\left(M_{0}\right)=\mathfrak{a} \alpha$. Applying our induction assumption to $L_{0}$ and $M_{0}$, we find a canonical base $\left\{y_{2}, \cdots, y_{n}, z_{2}, \cdots, z_{n}\right\}$ of $L_{0}$ and elements $a_{2}, \cdots, a_{n}, b_{2}, \cdots, b_{n}$ of $F$ such that 


$$
\begin{aligned}
& L_{0}=\mathfrak{g} y_{2}+\cdots+\mathfrak{g} y_{n}+\mathfrak{a} z_{2}+\cdots+\mathfrak{a} z_{n}, \\
& M_{0}=\mathfrak{g} a_{2} y_{2}+\cdots+\mathfrak{g} a_{n} y_{n}+\mathfrak{a} b_{2} z_{2}+\cdots+\mathfrak{a} b_{n} z_{n}, \\
& \alpha=a_{2} b_{2}=\cdots=a_{n} b_{n}, \\
& \mathfrak{g} a_{2} \supset \cdots \supset \mathfrak{g} a_{n} \supset \mathfrak{g} b_{n} \supset \cdots \supset \mathfrak{g} b_{2} .
\end{aligned}
$$

As $L_{0} \supset M_{0}$, we have $\mathrm{g} \supset \mathrm{g} a_{2}$, so that $\mathrm{g} b_{2} \supset \mathrm{g} \alpha$. Putting $a_{1}=1$ and $b_{1}=\alpha$, we obtain our assertion for $L$ and $M$.

Proposition 1.6. Let $L$ be a maximal lattice in $W$. Let $\left\{u_{1}, \cdots, u_{n}, v_{1}, \cdots, v_{n}\right\}$ be a canonical base of $L$. Denote by $\Gamma^{0}$ the subgroup of $G^{0}(W, g)$ consisting of elements $r$ of $G^{0}(W, g)$ such that $L r=L$, and by $\Delta$ the set of elements $\sigma$ of $G(W, g)$ such that $u_{i} \sigma=a_{i} u_{i}, v_{i} \sigma=b_{i} v_{i}$ for $1 \leqq i \leqq n$ with elements $a_{i}, b_{i}$ of $F$ and $\mathfrak{g} \alpha_{1} \supset \cdots$ $\supset \mathrm{g} a_{n} \supset \mathrm{g} b_{n} \supset \cdots \supset \mathrm{g} b_{1}$. Then we have $G(W, g)=\Gamma^{0} \cdot \Delta \cdot \Gamma^{0}$.

Proof. Let $\sigma$ be an element of $G(W, g)$. Put $M=L \sigma, \alpha=N(\sigma)$, and apply Proposition 1.5 to this $\{L, M, \alpha\}$. Then we get a canonical base $\left\{y_{i}, z_{i}\right\}$ of $L$ and elements $a_{i}, b_{i}$ of $F$ with the properties of that proposition. Define two. elements $\gamma$ and $\tau$ of $E(W)$ by $u_{i} r=y_{i}, v_{i} r=z_{i}, u_{i} \tau=a_{i} u_{i}, v_{i} \tau=b_{i} v_{i}$. We see easily that $\gamma \in \Gamma^{0}$ and $\tau \in \Delta, N(\tau)=\alpha$. Further we have $L \tau \gamma=L \sigma$. Hence if we put $\varepsilon \tau \gamma=\sigma$, we have $L \varepsilon=L, \varepsilon \in G(W, g), N(\varepsilon)=1$, so that $\varepsilon \in \Gamma^{0}$. It follows. that $\sigma=\varepsilon \tau \gamma \in \Gamma^{0} \cdot \Delta \cdot \Gamma^{0}$. Our proposition is thereby proved.

\section{$\S 2$. Hermitian forms over a quaternion algebra.}

2.1. Quaternion algebras. By a quaternion algebra over a field $F$, we understand a central simple algebra $A$ over $F$ such that $[A: F]=4$. Every quaternion algebra $A$ over $F$ has an involution $a \rightarrow a^{\prime}$, which is uniquely determined by the property that $(X-a)\left(X-a^{\prime}\right)$ is the principal polynomial of $a$ over $F$. We call it the canonical involution of $A$ and always denote it by $a \rightarrow a^{\prime}$. For every $a \in A$, we put

$$
N(a)=a \alpha^{\prime}, \quad \operatorname{Tr}(a)=a+\alpha^{\prime} .
$$

If $A$ is not a division algebra, $A$ is isomorphic to $M_{2}(F)$; and for every $a \in M_{2}(F), N(a)$ is just the determinant of $a$ and $\operatorname{Tr}(\alpha)$ is the trace of $\alpha$. Hereafter we assume that the characteristic of $F$ is different from 2. Then, for an element $a$ of $A$, we have $a=a^{\prime}$ if and only if $a \in F$.

If $F$ is the quotient field of a Dedekind domain $g$, we can develop idealtheory in $A$. Here we recall only the definition of different and norm of ideals. Let $\mathfrak{D}$ be a maximal order in $A$. The different $\mathfrak{D}=\mathfrak{D}(\mathfrak{D} / \mathfrak{g})$ of $\mathfrak{o}$ with respect to $g$ is the integral two-sided $\mathfrak{D}$-ideal defined by

$$
\mathfrak{D}^{-1}=\{x \in A \mid \operatorname{Tr}(x \mathfrak{D}) \subset \mathfrak{g}\} \text {. }
$$

Let $\mathfrak{a}$ be a right (resp. left) $\mathfrak{D}$-ideal. We denote by $N(\mathfrak{a})$ the g-ideal generated 
by the elements $N(a)$ for $a \in \mathfrak{a}$. If we put $\mathfrak{a}^{\prime}=\left\{x^{\prime} \mid x \in \mathfrak{a}\right\}$, then $\mathfrak{a}^{\prime} \mathfrak{a}=N(\mathfrak{a}) \mathfrak{b}$. (resp. $\left.\mathfrak{a} \mathfrak{a}^{\prime}=N(\mathfrak{a}) \mathfrak{o}\right)$.

2.2. $Q$-hermitian forms. Let $A$ be a quaternion algebra over a field $F$. By an $A$-space of dimension $n$, we understand a left $A$-module $V$ isomorphic to the product of $n$ copies of $A$; and we put $n=\operatorname{dim}_{A} V$. We call a set of elements $\left\{x_{1}, \cdots, x_{n}\right\}$ of $V$ a base of $V$ over $A$ if $V=A x_{1}+\cdots+A x_{n}$.

Let $V$ be an $A$-space of dimension $n$. We understand by a $Q$-hermitian form on $V$ an $F$-bilinear mapping $f$ of $V \times V$ into $A$ satisfying

$$
f(a x, y)=a f(x, y), \quad f(x, y)^{\prime}=f(y, x)
$$

for $a \in A, x \in V, y \in V$. We call $f$ non-degenerate if $f(x, V)=\{0\}$ implies $x=0$.

Proposition 2.1. Let $A$ be a quaternion algebra over a field $F$ and $V$ be an A-space of dimension $n$. For every $Q$-hermitian form $f(x, y)$ on $V$, there exists a base $\left\{x_{1}, \cdots, x_{n}\right\}$ of $V$ over $A$ such that $f\left(x_{i}, x_{j}\right)=\alpha_{i} \delta_{i j}$ for $1 \leqq i \leqq n, 1 \leqq j \leqq n$ with $\alpha_{i} \in F$. Moreover, suppose that $f$ is non-degenerate and $A$ satisfies the following condition:

(D) For every $\alpha \in F$, there exists an element a of $A$ such that $N(a)=\alpha$. Then there exists a base $\left\{y_{1}, \cdots, y_{n}\right\}$ of $V$ over $A$ such that $f\left(y_{i}, y_{j}\right)=\delta_{i j}$.

This is well-known and in fact easily proved. If $A=M_{2}(F)$, the condition (D) is clearly satisfied.

Let $V$ be an $A$-space. We denote by $E(V, A)$ the ring of all $F$-linear mappings $\sigma$ of $V$ into itself satisfying $(a x) \sigma=a(x \sigma)$ for every $a \in A, x \in V$, and by $G L(V, A)$ the group of regular elements of $E(V, A)$. Let $f$ be a nondegenerate $Q$-hermitian form on $V$. We denote by $G(V, f)$ the subgroup of $G L(V, A)$ consisting of the elements $\sigma$ for which there exists a number $N(\sigma)$. of $F$ such that $f(x \sigma, y \sigma)=N(\sigma) f(x, y)$ for every $x \in V, y \in V$; and put $G^{0}(V, f)$. $=\{\sigma \in G(V, f) \mid N(\sigma)=1\} . \quad G^{0}(V, f)$ is clearly a normal subgroup of $G(V, f)$. If $\xi$ is a non-zero element of $F$, we have $f(\xi x, \xi y)=\xi^{2} f(x, y)$; so we often consider $\xi$ as an element of $G(V, f)$. If $\operatorname{dim}_{A} V=1, E(V, A)$ is isomorphic to $A$, and $G(V, f)$ is isomorphic to the group of regular elements of $A$; for every $\sigma$ $\in G(V, f), N(\sigma)$ coincides with $N(\sigma)$ of $\sigma$ considered as an element of $A$.

Fix a base $\left\{x_{1}, \cdots, x_{n}\right\}$ of $V$ over $A$. Every element $\sigma$ of $E(V, A)$ is represented by a matrix $\left(s_{i j}\right)$ of $M_{n}(A)$ with respect to $\left\{x_{i}\right\}$ :

$$
x_{i} \sigma=\sum_{j=1}^{n} s_{i j} x_{j} \quad(1 \leqq i \leqq n) .
$$

For every element $S=\left(s_{i j}\right)$ of $M_{n}(A)$, we put $S^{\prime}=\left(t_{i j}\right)$ with $t_{i j}=s_{j i}{ }^{\prime}$. Then $S \rightarrow S^{\prime}$ is an involution of $M_{n}(A)$. Let $f(x, y)$ be a $Q$-hermitian form on $V$. Define an element $H=\left(h_{i j}\right)$ of $M_{n}(A)$ by $h_{i j}=f\left(x_{i}, x_{j}\right)$. Then we have $H^{\prime}=H$. An element $\sigma$ of $G L(V, A)$ belongs to $G(V, f)$ if and only if we have $S H S^{\prime}=\alpha H$ with $\alpha \in F$ for the matrix $S$ corresponding to $\sigma$; and then we have $N(\sigma)=\alpha$. 
2.3. Elementary theory of maximal lattices. Let $\mathfrak{g}$ be a Dedekind domain and $F$ the quotient field of g. Let $A$ be a quaternion algebra over $F$ and $V$ an $A$-space of dimension $n$. Take a non-degenerate $Q$-hermitian form $f$ on $V$. Let $L$ be a $\mathfrak{g}$-lattice in $V$. Put $\mathfrak{b}=\{a \in A \mid a L \subset L\}$. Then $\mathfrak{b}$ is an order in $A$. We call $\mathfrak{D}$ the order of $L$ and say that $L$ is normal if $\mathfrak{v}$ is a maximal order in $A$. Assume that $L$ is normal. We denote by $N_{f}(L)$ the two-sided $\mathfrak{D}$-ideal generated by the elements $f(x, y)$ for $x \in L, y \in L$, and call $N_{f}(L)$ the norm of $L$ with respect to $f$. We denote $N_{f}(L)$ simply by $N(L)$ when we fix $f$ and there is no fear of confusion.

Now, for every prime ideal $\mathfrak{p}$ of $\mathfrak{g}$, consider the $\mathfrak{p}$-completion $F_{\mathfrak{p}}$ and $\mathfrak{g}_{\mathfrak{p}}$ of

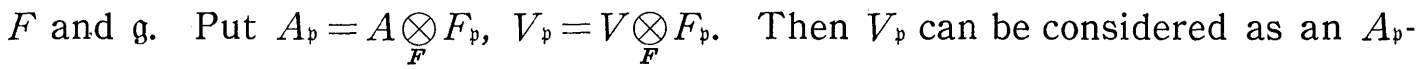
space of dimension $n$ in a natural manner. Further $f$ is uniquely extended to a non-degenerate $Q$-hermitian form on $V_{\mathfrak{p}}$, which we denote again by $f$. The following proposition is an easy consequence of our definition.

PROposition 2.2. Let $L$ be a $\mathfrak{g}$-lattice in $V$. If $\mathfrak{D}$ is the order of $L$, then $\mathfrak{D}_{\mathfrak{p}}\left(=\mathfrak{g}_{\mathfrak{p}} \mathfrak{D}\right)$ is the order of $L_{\mathfrak{p}}\left(=\mathfrak{g}_{\mathfrak{p}} L\right) . \quad L$ is normal if and only if $L_{\mathfrak{p}}$ is normal for every prime ideal $\mathfrak{p}$ of $\mathfrak{g}$. If $L$ is normal, we have $N(L)_{\mathfrak{p}}=N\left(L_{p}\right)$.

Let $L$ be a normal lattice in $V$ and $\mathfrak{o}$ the order of $L$. We call $L$ maximal (with respect to $f$ ) if $L$ is a maximal one among the normal lattices with the same order $\mathfrak{b}$ and the same norm $N(L)$.

Proposition 2.3. Let $L$ be a g-lattice in $V$ and $\sigma$ an element of $G(V, f)$. Then $L \sigma$ is a g-lattice in $V$ with the same order as $L$. If $L$ is normal, so is $L \sigma$; and we have $N(L \sigma)=N(L) N(\sigma)$. Moreover, if $L$ is maximal, so is $L \sigma$.

This is also an easy consequence of definition. Further, by Lemma 1.1 and Proposition 2.2, we obtain

Proposition 2.4. A normal $\mathrm{g}$-lattice in $V$ is maximal if and only if $L_{p}$ is maximal for every prime ideal $\mathfrak{p}$ of $\mathfrak{g}$.

Hereafter, we call a normal maximal $g$-lattice in $V$ simply a maximal lattice in $V$.

Proposition 2.5. Let $L$ be a normal g-lattice in $V$ with the order $\mathrm{D}$. Let $\mathfrak{a}$ be a right $\mathfrak{p}$-ideal and $\mathfrak{p}_{1}$ the left order of $\mathfrak{a}$. Then $a L$ is a normal $\mathrm{g}$-lattice in $V$ with the order $\mathfrak{b}_{1}$, and $N(\mathfrak{a} L)=\mathfrak{a} N(L) \mathfrak{a}^{-1} \cdot N(\mathfrak{a})$. Moreover, if $L$ is maximal, so is $a L$.

Proof. The first assertion is clear. Let $x=\sum_{i} a_{i} x_{i}$ and $y=\sum_{j} b_{j} y_{j}$ be elements of $\mathfrak{a} L$ where $a_{i}, b_{j} \in \mathfrak{a}$ and $x_{i}, y_{j} \in L$. Then we have $f(x, y)=\sum_{i, j} a_{i} f\left(x_{i}, y_{j}\right) b_{j}^{\prime}$ $\in \mathfrak{a} N(L) \mathfrak{a}^{\prime} . \quad$ As $\mathfrak{a} \mathfrak{a}^{\prime}=\mathfrak{p}_{1} N(\mathfrak{a})$, we get $\mathfrak{a} N(L) \mathfrak{a}^{\prime}=\mathfrak{a} N(L) \mathfrak{a}^{-1} \cdot N(\mathfrak{a})$. Therefore we obtain $N(\mathfrak{a} L) \subset \mathfrak{a} N(L) \mathfrak{a}^{-1} \cdot N(\mathfrak{a})$. Substituting $\mathfrak{a}^{-1}$ and $\mathfrak{a} L$ for $\mathfrak{a}$ and $L$, we get the inverse inclusion, so that the equality $N(\mathfrak{a} L)=\mathfrak{a} N(L) \mathfrak{a}^{-1} N(\mathfrak{a})$ holds. The last assertion follows easily from this relation.

Proposition 2.6. Let $\left\{x_{1}, \cdots, x_{n}\right\}$ be a base of $V$ over $A$ such that $f\left(x_{i}, x_{j}\right)$ 
$=\alpha_{i} \delta_{i j}$ with $\alpha_{i} \in F . \quad$ Let $\mathfrak{v}$ be a maximal order in $A$ and $\mathfrak{b}_{1}, \cdots, \mathfrak{b}_{n}$ be left $\mathfrak{b}$-ideals such that $\alpha_{1} N\left(\mathfrak{b}_{1}\right)=\cdots=\alpha_{n} N\left(\mathfrak{b}_{n}\right)$. Then $L=\mathfrak{b}_{1} x_{1}+\cdots+\mathfrak{b}_{n} x_{n}$ is a maximal lattice with the order $\mathfrak{D}$ and $N(L)=\alpha_{1} N\left(\mathfrak{b}_{1}\right)$.

PRoof. It is clear that $L$ is a $\mathfrak{g}$-lattice in $V$ with the order $\mathfrak{p}$, and $N(L)$ $=\alpha_{i} N\left(\mathfrak{b}_{i}\right)$. Let $M$ be a $\mathfrak{g}$-lattice with the order $\mathfrak{v}$ such that $M \supset L$ and $N(M)$ $=N(L)$. Let $y=\sum_{i=1}^{n} b_{i} x_{i}$ be an element of $M$ with $b_{i} \in A$. We have $b_{i} \alpha_{i} \mathfrak{b}_{i}^{\prime}$ $=f\left(y, \mathfrak{b}_{i} x_{i}\right) \subset N(M)=N(L)=\alpha_{i} \mathfrak{b}_{i} \mathfrak{b}_{i}^{\prime}$, so that $b_{i} \in \mathfrak{b}_{i}$. This implies $y \in L$ and hence $M=L$. Therefore $L$ is maximal.

Proposition 2.7. Let $L$ and $M$ be maximal lattices in $V$ with the same order. Let $\mathfrak{a}$ be a g-ideal. If $L \supset M$ and $N(M) \supset \mathfrak{a} N(L)$, then $M \supset \mathfrak{a} L$.

Proof. As $\mathfrak{a} N(L) \subset N(M) \subset N(L), \mathfrak{a}$ is an integral ideal. Put $K=M+\mathfrak{a} L$. Then $K$ is a $g$-lattice in $V$ with the same order as $L$. We have $f(K, K)$ $\subset f(M, M)+\mathfrak{a} f(M, L)+\mathfrak{a} f(L, M)+\mathfrak{a}^{2} f(L, L) \subset N(M)$, so that $N(K)=N(M)$. By the maximality of $M$, we must have $K=M$, and hence $a L \subset M$.

Proposition 2.8. Let $M$ be a normal g-lattice in $V$. Then there exists a maximal lattice $L$, with the same order as $M$, such that $N(L)=N(M)$ and $L \supset M$.

Proof. Let $\mathfrak{D}$ be the order of $M$. Take a base $\left\{x_{1}, \cdots, x_{n}\right\}$ of $V$ over $A$ such that $x_{i} \in M$ for every $i$. Put $K=\left\{y \mid f\left(y, x_{i}\right) \in N(M)\right.$ for every $\left.i\right\}$. It is easy to see that $K$ is a g-lattice in $V$ with order $\mathfrak{D}$. Now, let $L$ be a g-lattice in $V$ with order $\mathfrak{D}$ such that $L \supset M$ and $N(L)=N(M)$. If $y \in L$, we have $f\left(y, x_{i}\right)$ $\in N(L)=N(M)$, so that $y \in K$. Hence $L$ is contained in $K$. As $K$ is a $g$-lattice, the ascending chain condition holds for the $g$-lattices contained in $K$. Therefore, we can find a maximal one among the lattices containing $M$, with order $\mathfrak{b}$ and with norm $N(M)$. This proves our proposition.

2.4. The relation between alternating form and $\boldsymbol{Q}$-hermitian form. We now consider the case $A=M_{2}(F)$ for an arbitrary field $F$. Let $e_{i j} \quad(i=1,2$; $j=1,2)$ be the matrix units of $A$. We note that $e_{11}^{\prime}=e_{22}, e_{12}^{\prime}=-e_{12}, e_{21}^{\prime}=-e_{21}$. Let $V$ be an $A$-space of dimension $n$ and $f$ a $Q$-hermitian form on $V$. Put $W_{i}=e_{i i} V$ for $i=1,2$. Then $W_{i}$ is a vector space over $F$ of dimension $n$ for $i=1,2$; and $V$ is the direct sum of $W_{1}$ and $W_{2}$. If $x, y \in W_{1}$, we have $f(x, y)$ $=f\left(e_{11} x, e_{11} y\right)=e_{11} f(x, y) e_{22} \in F e_{12}$. Hence we can define an $F$-bilinear mapping $g$ of $W_{1} \times W_{1}$ into $F$ by

$$
f(x, y)=g(x, y) e_{12} .
$$

As $e_{12}^{\prime}=-e_{12}$, we have $g(y, x) e_{12}=f(y, x)=f(x, y)^{\prime}=-g(x, y) e_{12}$, so that $g$ is an alternating form on $W_{1}$. If $\sigma \in E(V, A)$, we have $W_{1} \sigma \subset W_{1}$; so the restriction of $\sigma$ to $W_{1}$ gives rise to an element of $E\left(W_{1}\right)$, which we denote by $\sigma_{1}$.

Proposition 2.9. Notation being as above, the mapping $\sigma \rightarrow \sigma_{1}$ gives an isomorphism of $E(V, A)$ and $G L(V, A)$ onto $E\left(W_{1}\right)$ and $G L\left(W_{1}\right)$, respectively. Moreover, suppose that $f$ is non-degenerate. Then $g$ is non-degenerate; and $\sigma \rightarrow \sigma_{1}$ 
gives an isomorphism of $G(V, f)$ and $G^{0}(V, f)$ onto $G\left(W_{1}, g\right)$ and $G^{0}\left(W_{1}, g\right)$, respectively; and further we have $N(\sigma)=N\left(\sigma_{1}\right)$ for $\sigma \in G(V, f)$.

This can be proved in an almost straightforward way. By Proposition 2.1 , there exists a base $\left\{x_{1}, \cdots, x_{n}\right\}$ of $V$ over $A$ such that $f\left(x_{i}, x_{j}\right)=\delta_{i j}$. Using matricial representation with respect to this base, the mapping $\sigma \rightarrow \sigma_{1}$ is given explicitly as follows. First note that $\left\{e_{11} x_{i}, e_{12} x_{i}(1 \leqq i \leqq n)\right\}$ is a base of $W_{1}$ over $F$. Let $\sigma$ be an element of $E(V, A)$ and $S=\left(s_{i j}\right)$ the element of $M_{n}(A)$ determined by (1) of $\S 2.2$. Put $e_{11} x_{i}=y_{i}, e_{12} x_{i}=z_{i}$ and $s_{i j}=\left(\begin{array}{ll}a_{i j} & b_{i j} \\ c_{i j} & d_{i j}\end{array}\right)$ with $a_{i j}, b_{i j}, c_{i j}, d_{i j}$ in $F$. Then we have $y_{i} \sigma=\sum_{j=1}^{n} a_{i j} y_{j}+\sum_{j=1}^{n} b_{i j} z_{j}, z_{i} \sigma=\sum_{j=1}^{n} c_{i j} y_{j}+\sum_{j=1}^{n} d_{i j} z_{j}$. Now define an isomorphism $\iota$ of $M_{n}(A)$ onto $M_{2 n}(F)$ by $\iota\left(s_{i j}\right)=\left(\begin{array}{cc}\left(a_{i j}\right) & \left(b_{i j}\right) \\ \left(c_{i j}\right) & \left(d_{i j}\right.\end{array}\right)$. Then $\sigma_{1}$ is represented by $\iota(S)$ with respect to the base $\left\{y_{i}, z_{i}\right\}$. As $f\left(x_{i}, x_{j}\right)$ $=\delta_{i j}$, we get $g\left(y_{i}, y_{j}\right)=g\left(z_{i}, z_{j}\right)=0, g\left(y_{i}, z_{j}\right)=-\delta_{i j}$. Put $J=\left(\begin{array}{cc}0 & 1_{n} \\ -1_{n} & 0\end{array}\right)$. Then we have $\iota\left(S^{\prime}\right)=\left(\begin{array}{rr}{ }^{t}\left(d_{i j}\right) & -{ }^{t}\left(b_{i j}\right) \\ -{ }^{t}\left(c_{i j}\right) & { }^{t}\left(a_{i j}\right)\end{array}\right)=J \cdot{ }^{t} \iota(S) J^{-1}$. Therefore, if $S S^{\prime}=\alpha 1_{n}$ with $\alpha \in F$, we have ${ }_{l}(S) J{ }^{t}{ }_{l}(S)=\alpha J$. This will give a 'non-intrinsic' proof of Proposition 2.8.

2.5. Paraphrase of the result of $\S 1$. The notation $A=M_{2}(F), V, f, W_{i}$, $g$ being as in $\S 2.4$, suppose that $F$ is the quotient field of a Dedekind domain g. For every $\mathfrak{g}$-ideal $\mathfrak{c}$, put $\mathfrak{b}(\mathfrak{c})=\mathfrak{g} e_{11}+\mathfrak{c} e_{12}+\mathfrak{c}^{-1} e_{21}+\mathfrak{g} e_{22}=\left(\begin{array}{ll}\mathfrak{g} & \mathfrak{c} \\ \mathfrak{c}^{-1} & \mathfrak{g}\end{array}\right)$. Then, $\mathfrak{b}(\mathfrak{c})$ is a maximal order in $A$; and for every maximal order $\mathfrak{D}$ in $A$, there exist an element $a$ of $A$ and a $\mathfrak{g}$-ideal $\mathfrak{c}$ such that $\alpha \mathfrak{a} a^{-1}=\mathfrak{p}(\mathfrak{c})$. Fix a $\mathfrak{g}$-ideal $\mathfrak{c}$ and put $\mathfrak{D}=\mathfrak{D}(\mathfrak{c})$. Let $L$ be a $\mathfrak{g}$-lattice in $V$ with the order $\mathfrak{o}$. Put $e_{i i} L=M_{i}$. Then we have $M_{i}=L \cap W_{i}, L=M_{1}+M_{2}$; and $M_{i}$ is a g-lattice in $W_{i}$. Further we have $M_{1}=\mathfrak{c}_{12} M_{2}, M_{2}=\mathfrak{c}^{-1} e_{21} M_{1}$. Now, by Proposition 1.3, there exist $\mathrm{g}$-ideals $\mathfrak{a}_{1}, \cdots, \mathfrak{a}_{n}$ and a base $\left\{y_{i}, z_{i}\right\}$ of $W_{1}$ over $F$ with the properties of that proposition for $M=M_{1}$. Then we have

$$
M_{2}=\mathfrak{c}^{-1} e_{21} y_{1}+\cdots+\mathfrak{c}^{-1} e_{21} y_{n}+\mathfrak{c}^{-1} \mathfrak{a}_{1} e_{21} z_{1}+\cdots+c^{-1} \mathfrak{a}_{n} e_{21} z_{n} .
$$

Put $x_{i}=y_{i}-e_{21} z_{i}, \mathfrak{b}_{i}=\mathfrak{g} e_{11}+\mathfrak{c} \mathfrak{a}_{i} e_{12}+\mathfrak{c}^{-1} e_{21}+\mathfrak{a}_{i} e_{22}=\left(\begin{array}{ll}\mathfrak{g} & \mathfrak{a}_{i} \\ \mathfrak{c}^{-1} & \mathfrak{c}^{-1} \mathfrak{a}_{i}\end{array}\right)$ for $1 \leqq i \leqq n$. Then the $\mathfrak{h}_{i}$ are left $\mathfrak{n}$-ideals; and we see easily $L=\mathfrak{b}_{1} x_{1}+\cdots+\mathfrak{b}_{n} x_{n}, \mathfrak{b}_{1} \supset \cdots \supset \mathfrak{b}_{n}$, $f\left(x_{i}, x_{j}\right)=\delta_{i j} . \quad$ Further we have $N(L)=N\left(\mathfrak{b}_{1}\right) \mathfrak{b}, N\left(\mathfrak{b}_{1}\right)=\mathfrak{c}^{-1} \mathfrak{a}_{1}=\mathfrak{c}^{-1} N_{g}\left(M_{1}\right)$. Therefore, if $L$ is maximal, we must have $\mathfrak{b}_{1}=\cdots=\mathfrak{b}_{n}$, and hence $\mathfrak{a}_{1}=\cdots=\mathfrak{a}_{n}$, so that $M_{1}$ is maximal with respect to $g$. Thus we obtain

Proposition 2.10. Let $F$ be the quotient field of a Dedekind domain $\mathfrak{g}$, and $A=M_{2}(F)$. Let $V$ be an A-space of dimension $n$ and $f$ be a non-degenerate $Q$ hermitian form on $V$. Let $L$ be a normal lattice in $V$ and $\mathfrak{D}$ the order of $L$. Then there exist left $\mathrm{D}$-ideals $\mathfrak{b}_{1}, \cdots, \mathfrak{b}_{n}$ and a base $\left\{x_{1}, \cdots, x_{n}\right\}$ of $V$ over $A$ such 
that

$$
\begin{gathered}
L=\mathfrak{b}_{1} x_{1}+\cdots+\mathfrak{b}_{n} x_{n}, \quad \mathfrak{b}_{1} \supset \cdots \supset \mathfrak{b}_{n}, \\
f\left(x_{i}, x_{j}\right)=\delta_{i j} ;
\end{gathered}
$$

and we have $N(L)=N\left(\mathfrak{b}_{1}\right)$ o. Moreover, if $L$ is maximal, $\mathfrak{b}_{1}=\cdots=\mathfrak{b}_{n}$.

Proposition 2.11. Notation being as in Proposition 2.10, let $L_{1}$ and $L_{2}$ be maximal lattices in $V$ with the same order $\mathfrak{D}$. If $L_{1} \sigma=L_{2}$ for an element $\sigma$ of $G(V, f)$, then $\alpha N\left(L_{1}\right)=N\left(L_{2}\right)$ for an element $\alpha$ of $F$. Conversely, if $\alpha N\left(L_{1}\right)=N\left(L_{2}\right)$ with $\alpha \in F$, we can find an element $\sigma$ of $G(V, f)$ such that $L_{1} \sigma=L_{2}, N(\sigma)=\alpha$.

Proof. The first assertion is obvious. Now suppose that $N\left(L_{2}\right)=\alpha N\left(L_{1}\right)$ with $\alpha \in F$. We may assume that $\mathfrak{D}=\mathfrak{b}(\mathfrak{c})$ for a $\mathfrak{g}$-ideal c. Put $M_{i}^{1}=e_{i i} L_{1}, M_{i}^{2}$ $=e_{i i} L_{2}$ for $i=1,2$. By the above consideration, $M_{1}^{1}$ and $M_{1}^{2}$ are maximal lattices in $W_{1}$, and $N_{g}\left(M_{1}^{1}\right) \alpha=N_{g}\left(M_{1}^{2}\right)$. By Proposition 1.4 and its proof, there exists an element $\sigma_{1}$ of $G\left(W_{1}, g\right)$ such that $M_{1}^{1} \sigma_{1}=M_{1}^{2}, N\left(\sigma_{1}\right)=\alpha$. Let $\sigma$ be an element of $G(V, f)$ corresponding to $\sigma_{1}$ by the mapping of Proposition 2.9. We get then $N(\sigma)=\alpha$, and $L_{1} \sigma=L_{2}$, since $L_{1}=\mathrm{o} M_{1}^{1}, L_{2}=\mathrm{o} M_{1}^{2}$. This completes our proof. We can also derive our proposition more directly from Proposition 2.10.

Proposition 2.12. Notation being as in Proposition 2.10, suppose that $\mathfrak{g}$ is a principal ideal domain. Put $\mathfrak{D}=M_{2}(\mathrm{~g})$. Let $L$ and $M$ be maximal lattices in $V$ with the order $\mathfrak{D}$. Let $\eta$ and $\alpha$ be elements of $F$ such that $N(L)=\eta \mathbb{0}$ and $N(M)$ $=\alpha N(L)$. Then there exist a base $\left\{x_{1}, \cdots, x_{n}\right\}$ of $V$ over $A$ and elements $a_{1}, \cdots, a_{n}$, $b_{1}, \cdots, b_{n}$ of $F$ such that

$$
\begin{aligned}
& f\left(x_{i}, x_{j}\right)=\eta \delta_{i j}, \\
& L=\mathfrak{D} x_{1}+\cdots+\mathfrak{o} x_{n}, \\
& M=\mathfrak{D} e_{1} x_{1}+\cdots+\mathfrak{D} e_{n} x_{n}, \quad e_{i}=\left(\begin{array}{cc}
a_{i} & 0 \\
0 & b_{i}
\end{array}\right) \quad(0 \leqq i \leqq n), \\
& \mathfrak{g} a_{1} \supset \cdots \supset \mathfrak{g} a_{n} \supset \mathfrak{g} b_{n} \supset \cdots \supset \mathfrak{g} b_{1}, \\
& \alpha=a_{1} b_{1}=\cdots=a_{n} b_{n} .
\end{aligned}
$$

Proof. Put $L_{1}=e_{11} L, M_{1}=e_{11} M$. Then $L_{1}$ and $M_{1}$ are maximal lattices in $W_{1}$ with respect to $g$, and $N\left(L_{1}\right)=g \eta, N\left(M_{1}\right)=g \alpha \eta$. Applying Proposition 1.5 to this $\left\{L_{1}, M_{1}, \alpha\right\}$, we obtain $\left\{y_{i}, z_{i}\right\}$ and $\left\{a_{i}, b_{i}\right\}$ with the properties of that proposition for $L_{1}$ and $M_{1}$. Put $x_{i}=y_{i}-e_{21} \eta z_{i}$. Then we can easily verify that $f\left(x_{i}, x_{j}\right)=\eta \delta_{i j}, L=\mathrm{D} x_{1}+\cdots+\mathfrak{D} x_{n}, M=\mathrm{D} e_{1} x_{1}+\cdots+\mathrm{D} e_{n} x_{n}$ with $e_{i}=\left(\begin{array}{cc}a_{i} & 0 \\ 0 & b_{i}\end{array}\right)$. This proves our proposition.

Notation being as in Proposition 2.12, we call $\left\{\mathrm{g} a_{1}, \cdots, \mathrm{g} a_{n}, \mathrm{~g} b_{1}, \cdots, \mathrm{g} b_{n}\right\}$ the set of elementary divisors of $M$ relative to $L$ and denote it by $\{L: M\}$. We get an assertion for $\{V, f\}$ which is a paraphrase of Proposition 1.6. Instead of stating it, we give the following proposition.

Proposition 2.13. Notation and assumption being as in Proposition 2.12, 
let $L, M, K$ be maximal lattices in $V$ with the order $\mathfrak{D}$. Then there exists an element $\sigma$ of $G^{0}(V, f)$ such that $L \sigma=L$ and $M \sigma=K$, if and only if $\{L: M\}$ $=\{L: K\}$.

Proof. The 'only if' part is clear. Put $N(L)=\mathrm{o} \eta, N(M)=\mathrm{p} \alpha \eta, N(K)$ $=\mathfrak{o} \beta \eta$ with $\alpha, \beta, \eta \in F$. By Proposition 2.12, we get a base $\left\{x_{i}\right\}$ of $V$ over $A$ and a set of elements $\left\{a_{i}, b_{i}\right\}$ of $F$ for $M$ with the properties of that proposition, and a base $\left\{u_{i}\right\}$ of $V$ over $A$ and a set of elements $\left\{c_{i}, d_{i}\right\}$ of $F$ with the corresponding properties for $K$. If $\{L: M\}=\{L: K\}$, we have $g a_{i}=\mathrm{g} c_{i}$, $\mathrm{g} b_{i}=\mathrm{g} d_{i}$, so that $\mathrm{g} \alpha=\mathrm{g} \beta$. Hence we may put $\alpha=\beta$. We have then $a_{i} b_{i}=c_{i} d_{i}$. Let $\varepsilon_{i}$, for each $i$, be a unit of $g$ such that $\varepsilon_{i} a_{i}=c_{i}$; then we have $\varepsilon_{i}^{-1} b_{i}=d_{i}$. Define an element $\sigma$ of $E(V, A)$ by $x_{i} \sigma=\left(\begin{array}{cc}\varepsilon_{i} & 0 \\ 0 & \varepsilon_{i}^{-1}\end{array}\right) u_{i}$. Then we see easily $\sigma$ $\in G^{0}(V, f), L \sigma=L, M \sigma=K$. This proves the 'if' part.

REMARK. Notation being as in Proposition 2.13, suppose that $L \supset M$, $L \supset K$. Then the following three conditions are equivalent to each other.

i) $\{L: M\}=\{L: K\}$.

ii) $L / M$ and $L / K$ are isomorphic as $g$-modules.

iii) $L / M$ and $L / K$ are isomorphic as $p$-modules.

\section{$\S 3$. Local theory of $Q$-hermitian forms.}

3.1. Quaternion algebras over local fields. By a $\mathfrak{p}$-adic number field, we understand a finite extension of the $p$-adic number field, for any prime number $p$. In this $\S 3, F_{\mathfrak{p}}, \mathfrak{g}_{\mathfrak{p}}, \mathfrak{p}$ denote respectively a $\mathfrak{p}$-adic number field, the ring of $\mathfrak{p}$-integers in $F_{\mathfrak{p}}$, the maximal ideal of $\mathfrak{g}_{\mathfrak{p}}$. It is well-known that there exist, up to isomorphism, only two quaternion algebras over $F_{\mathfrak{p}}$, the matric algebra $M_{2}\left(F_{\mathfrak{p}}\right)$ and a division algebra. The latter is written as $(B, S, \pi)=B+B u$ in the usual notation of cyclic algebra, where $B$ is the unique unramified quadratic extension of $F_{\mathfrak{p}}, S$ is the Frobenius automorphism of $B$ over $F_{\mathfrak{p}}, \pi$ is a prime element of $F_{\mathfrak{p}}$, and $u \beta u^{-1}=\beta^{s}$ for $\beta \in B, u^{2}=\pi$. We denote this division quaternion algebra over $F_{\mathfrak{p}}$ by $D_{\mathfrak{p}}$.

For every maximal order $\mathfrak{o}_{\mathfrak{p}}$ in $M_{2}\left(F_{\mathfrak{p}}\right)$, there exists an element $w$ such that $w_{\mathrm{o}_{p}} w^{-1}=M_{2}\left(\mathrm{~g}_{\mathrm{p}}\right) . \quad$ Every one-sided $\mathfrak{p}_{p}$-ideal is principal. Every two-sided $\mathfrak{p}_{p}$-ideal $\mathfrak{a}_{\mathfrak{p}}$ is written in the form $\mathfrak{a}_{\mathfrak{p}}=\mathfrak{p}^{\nu} \mathfrak{D}_{\mathfrak{p}}$ with $\nu \in \boldsymbol{Z}$, and conversely. Further $\mathfrak{D}\left(\mathfrak{b}_{\mathfrak{p}} / \mathfrak{g}_{\mathfrak{p}}\right)$ $=\mathfrak{o}_{\mathfrak{p}}$. As for $D_{\mathfrak{p}}$, it has only one maximal order $\mathfrak{b}_{\mathfrak{p}}=\left\{x \in D_{\mathfrak{p}} \mid N(x) \in \mathfrak{g}_{\mathfrak{p}}\right\}$; and every one-sided $\mathfrak{D}_{\mathcal{p}}$-ideal is principal and equal to a power of the maximal ideal $\mathfrak{P}$, so that it is a two-sided $\mathfrak{p}_{\mathfrak{p}}$-ideal. We have $\mathfrak{P}^{2}=\mathfrak{p} \mathfrak{o}_{\mathfrak{p}}, \mathfrak{P}=\mathfrak{D}\left(\mathfrak{o}_{\mathfrak{p}} / \mathfrak{g}_{\mathfrak{p}}\right)$.

PROPOSITION 3.1. Let $A_{\mathfrak{p}}$ be a quaternion algebra over $F_{\mathfrak{p}}$ and $\mathfrak{D}_{\mathfrak{p}}$ a maximal order in $A_{\mathfrak{p}}$. For every two-sided $\mathfrak{a}_{\mathfrak{p}}$-ideal $\mathfrak{a}_{\mathfrak{p}}$, we have $\operatorname{Tr}\left(\mathfrak{a}_{\mathfrak{p}}\right)=\mathfrak{a}_{\mathfrak{p}} \cap F_{\mathfrak{p}}$.

Proof. This is clear if $A_{p}=M_{2}\left(F_{\mathfrak{p}}\right)$. Therefore suppose that $A_{\mathfrak{p}}=D_{p}$. Then every $\mathfrak{D}_{\mathfrak{p}}$-ideal $\mathfrak{a}_{\mathfrak{p}}$ is written in the form $\mathfrak{a}_{\mathfrak{p}}=\mathfrak{P}^{e} \cdot\left(\mathfrak{a}_{\mathfrak{p}} \cap F_{\mathfrak{p}}\right)$ with $e=0$ or 
-1. We have $\operatorname{Tr}\left(\mathfrak{a}_{\mathfrak{p}}\right)=\operatorname{Tr}\left(\mathfrak{P}^{e}\right) \cdot\left(\mathfrak{a}_{\mathfrak{p}} \cap F_{\mathfrak{p}}\right)$ and $\operatorname{Tr}\left(\mathfrak{o}_{\mathfrak{p}}\right) \subset \operatorname{Tr}\left(\mathfrak{P}^{e}\right) \subset \operatorname{Tr}\left(\mathfrak{P}^{-1}\right) \subset \mathfrak{g}_{\mathfrak{p}_{\mathfrak{k}}}$ Therefore, it is sufficient to prove $\operatorname{Tr}\left(\mathfrak{o}_{\mathfrak{p}}\right)=\mathfrak{g}_{\mathfrak{p}}$. As $\operatorname{Tr}\left(\mathfrak{o}_{\mathfrak{p}}\right)$ is an integral $\mathfrak{g}_{\mathfrak{p}}$-ideal, there exists an integer $\nu \geqq 0$ such that $\operatorname{Tr}\left(\mathfrak{p}_{\mathfrak{p}}\right)=\mathfrak{p}^{\nu} \mathfrak{g}_{\mathfrak{p}}$. We get then $\operatorname{Tr}\left(\mathfrak{p}^{-\nu} \mathfrak{p}_{\mathfrak{p}}\right) \subset \mathfrak{g}_{\mathfrak{p}}$, so that $\mathfrak{p}^{-\nu} \mathfrak{D}_{\mathfrak{p}} \subset \mathfrak{D}\left(\mathfrak{d}_{\mathfrak{p}} / \mathfrak{g}_{\mathfrak{p}}\right)^{-1}=\mathfrak{P}^{-1}$, which implies $\nu=0$, since $\mathfrak{P}^{2}=\mathfrak{p}_{\mathfrak{p}}$. This completes our proof.

Proposition 3.2. $A_{p}$ and $\mathfrak{n}_{p}$ being as in Proposition 3.1, let $\beta$ be a non-zero element of $\mathfrak{g}_{\mathfrak{p}}$ and $a$ an element of $\mathfrak{o}_{\mathfrak{p}}$ such that $\beta^{-1} N(\alpha) \equiv 1 \bmod \mathfrak{p}^{\lambda}$ where $\lambda$ is $a$ positive integer. Then there exists an element $b$ of $\mathfrak{o}_{p}$ such that $N(b)=\beta_{\text {r }}$ $b \equiv a \bmod \mathfrak{p}^{\lambda} \mathfrak{D}_{\mathfrak{p}}$.

Proof. We first consider the case $A_{\mathfrak{p}}=M_{2}\left(F_{\mathfrak{p}}\right)$. We may then put $\mathfrak{p}_{\mathfrak{p}}$. $=M_{2}\left(\mathfrak{g}_{\mathfrak{p}}\right)$. Let $\pi$ be a prime element of $F$. We can find elements $x, y$ of $\mathfrak{p}_{\mathfrak{p}}$ such that $N(x)=N(y)=1, x a y=\left(\begin{array}{cc}\pi^{\prime \prime} \varepsilon & 0 \\ 0 & \pi^{\nu} \eta\end{array}\right)$ where $0 \leqq \mu \leqq \nu$, and $\varepsilon, \eta$ are units of $g_{p}$. As $\beta^{-1} N(a) \equiv 1 \bmod \mathfrak{p}^{\lambda}$, we have $\beta=\pi^{\mu+\nu} \delta$ with a unit $\delta$ of $\mathfrak{g}_{\mathfrak{p}}$, and $\delta \equiv \varepsilon \eta \bmod \mathfrak{p}^{\lambda}$. Put $b=x^{-1}\left(\begin{array}{cc}\pi^{\mu} \varepsilon & 0 \\ 0 & \pi^{\nu} \varepsilon^{-1} \delta\end{array}\right) y^{-1}$. As $\varepsilon^{-1} \delta \equiv \eta \bmod \mathfrak{p}^{\lambda}$, and as $x^{-1}, y^{-1} \in \mathfrak{p}_{\mathfrak{p}}$, we have $b \equiv a \bmod \mathfrak{p}^{\lambda}$, and clearly $N(b)=\pi^{\mu+\nu} \delta=\beta$. This proves our assertion for $A_{p}$ $=M_{2}\left(F_{\mathfrak{p}}\right)$. Now put $A_{\mathfrak{p}}=D_{\mathfrak{p}}$. Let $\Pi$ be a prime element in $\mathfrak{o}_{\mathfrak{p}}$; put $N(\Pi)=\pi$; then $\pi$ is a prime element in $\mathfrak{g}_{\mathfrak{p}}$. Put $a=\Pi^{\nu} e$ with a unit $e$ of $\mathfrak{p}_{\mathfrak{p}}$. As $\beta^{-1} N(a)$. $\equiv 1 \bmod \mathfrak{p}^{\lambda}$, we have $\beta=\pi^{\nu} \varepsilon$ with a unit $\varepsilon$ of $\mathfrak{g}_{\mathfrak{p}}$, and $N(e) \equiv \varepsilon \bmod \mathfrak{p}^{\lambda}$. Now we construct inductively a sequence $\left\{e_{0}, e_{1}, \cdots, e_{n}, \cdots\right\}$ of units of $\mathfrak{p}_{p}$ such that $e_{0}$. $=e, N\left(e_{n}\right) \equiv \varepsilon \bmod \mathfrak{p}^{\lambda+n}, e_{n+1} \equiv e_{n} \bmod \mathfrak{p}^{\lambda+n} \mathfrak{D}_{\mathfrak{p}} . \quad$ Assume that $e_{n}$ is already defined. Put $\varepsilon-N\left(e_{n}\right)=\pi^{\lambda+n} \cdot \gamma$ with $\gamma \in \mathfrak{g}_{\mathfrak{p}}$. By Proposition 3.1, we have $\operatorname{Tr}\left(e_{n}^{\prime} \mathfrak{b}_{\mathfrak{p}}\right)=\operatorname{Tr}\left(\mathfrak{p}_{\mathfrak{p}}\right)$ $=\mathfrak{g}_{\mathfrak{p}}$, so that there exists an element $d$ of $\mathfrak{o}_{\mathfrak{p}}$ such that $\operatorname{Tr}\left(e_{n}^{\prime} d\right)=r$. Put $e_{n+1}=e_{n}+\pi^{\lambda+n} d$. Then we have $N\left(e_{n+1}\right)=N\left(e_{n}\right)+\pi^{\lambda+n} \operatorname{Tr}\left(e_{n}^{\prime} d\right)+\pi^{2(\lambda+n)} N(d) \equiv \varepsilon$ $\bmod \mathfrak{p}^{\lambda+n+1}$. We get thus a sequence $\left\{e_{n}\right\}$ with the required property. As $e_{n+1} \equiv e_{n} \bmod \mathfrak{p}^{\lambda+n} \mathfrak{D}_{\mathfrak{p}}$, this converges to a unit $h$ of $\mathfrak{D}_{\mathfrak{p}}$, for which we have $N(h)$ $=\varepsilon, h \equiv e \bmod \mathfrak{p}^{\lambda} \mathfrak{D}_{\mathfrak{p}}$. Put $b=\Pi^{\nu} h$. Then we have $N(b)=\beta, b \equiv a \bmod \mathfrak{p}^{\lambda} \mathfrak{D}_{\mathfrak{p}}$. This. completes our proof.

Proposition 3.3. $A_{\mathfrak{p}}$ and $\mathfrak{D}_{\mathfrak{p}}$ being as in Proposition 3.1, let $\xi$ be an element of $g_{p} . \quad$ Then there exists an element $x$ of $\mathfrak{o}_{p}$ such that $N(x)=\xi$. In particular, $A_{\mathrm{p}}$ satisfies the condition (D) of Proposition 2.1.

PROOF. If $A_{\mathfrak{p}}=M_{2}\left(F_{\mathfrak{p}}\right)$, we may put $\mathfrak{o}_{\mathfrak{p}}=M_{2}\left(\mathfrak{g}_{\mathfrak{p}}\right)$, so that our assertion is. obvious. If $A_{\mathfrak{p}}=D_{\mathfrak{p}}$, it is well-known that any quadratic extension of $F_{\mathfrak{p}}$ is. isomorphic to a subfield of $D_{\mathfrak{p}}$, over $F_{\mathfrak{p}}$. Hence, for every $\xi \in F_{\mathfrak{p}}$, there exists an element $x$ of $D_{\mathfrak{p}}$ such that $N(x)=\xi$. If $\xi \in \mathfrak{g}_{\mathfrak{p}}$, we have $x \in \mathfrak{D}_{\mathfrak{p}}$ automatically. This completes our proof.

3.2. Canonical bases of maximal lattices. Let $A_{\mathfrak{p}}$ be a quaternion algebra over $F_{p}$, and $V_{p}$ be an $A_{p}$-space of dimension $n$. Take a non-degenerate $Q$ hermitian form $f$ on $V_{\mathfrak{p}}$. By Proposition 3.3 and Proposition 2.1, we see that, for every regular element $H=\left(h_{i j}\right)$ of $M_{n}\left(A_{\mathfrak{p}}\right)$ such that $H^{\prime}=H$, there exists 
a base $\left\{x_{1}, \cdots, x_{n}\right\}$ of $V_{\mathfrak{p}}$ over $A_{\mathfrak{p}}$ for which $f\left(x_{i}, x_{j}\right)=h_{i j}$. In particular we get

Proposition 3.4. $V_{\mathfrak{p}}$ has a base $\left\{x_{1}, \cdots, x_{m}, y_{1}, \cdots, y_{m}, z\right\}$ over $A_{\mathfrak{p}}$ such that

$$
\begin{aligned}
& f\left(x_{i}, x_{j}\right)=f\left(y_{i}, y_{j}\right)=f\left(x_{i}, z\right)=f\left(y_{i}, z\right)=0, \\
& f\left(x_{i}, y_{j}\right)=a \delta_{i j}, \quad f(z, z)=\beta \quad(1 \leqq i \leqq m, 1 \leqq j \leqq m),
\end{aligned}
$$

where $a$ is a regular element of $A_{\mathfrak{p}}, \beta$ is a non-zero element of $F_{\mathfrak{p}}$, the last member $z$ (and hence $\beta$ ) occurring only in the case where $n$ is odd.

We call a base $\left\{x_{1}, \cdots, x_{m}, y_{1}, \cdots, y_{m}, z\right\}$ of $V_{\mathfrak{p}}$ over $A_{\mathfrak{p}}$ with the property of the above proposition a canonical base of $V_{p}$. Proposition 3.4 implies that, if $n>1, V_{p}$ contains an element $x$ such that $f(x, x)=0, A_{p} x \cong A_{p}$.

Now we want to study the arithmetic of maximal lattices in $V_{p}$. If $A_{\text {p }}$ $=M_{2}\left(F_{\mathfrak{p}}\right)$, we can apply the theory of $\S 2.5$ and $\S 1$ to the present case, since $\mathfrak{g}_{\mathrm{p}}$ is a principal ideal domain; and this is sufficient for our later use. Therefore, we have only to consider the case $A_{p}=D_{p}$. From now on, until the end of this $\S 3.2, V_{p}$ is a $D_{p}$-space of dimension $n$, and $\mathfrak{o}_{\mathfrak{p}}$ denotes the unique maximal order in $D_{p}$.

Proposition 3.5. Let $L$ be a maximal lattice in $V_{p}$. Let a be an element of $D_{p}$ such that $N(L)=\mathfrak{o}_{\mathfrak{p}} a$, and $\beta$ be an element of $F_{\mathfrak{p}}$ such that $N(L) \cap F_{\mathfrak{p}}=\mathfrak{g}_{\mathfrak{p}} \beta$. Then there exists a canonical base $\left\{x_{1}, \cdots, x_{m}, y_{1}, \cdots, y_{m}, z\right\}$ of $V_{p}$ such that

$$
\begin{gathered}
L=\mathfrak{b}_{\mathfrak{p}} x_{1}+\mathfrak{o}_{\mathfrak{p}} y_{1}+\cdots+\mathfrak{p}_{\mathfrak{p}} x_{m}+\mathfrak{o}_{\mathfrak{p}} y_{m}+\mathfrak{o}_{\mathfrak{p}} z, \\
f\left(x_{i}, y_{j}\right)=a \delta_{i j}, \quad f(z, z)=\beta,
\end{gathered}
$$

where the term $\mathrm{o}_{\mathrm{p}} z$ and $\beta$ occur only when $n$ is odd. Conversely, let a be a regular element of $D_{p}$ and $\beta$ an element of $F_{\mathfrak{p}}$ such that $\left(\mathfrak{D}_{\mathfrak{p}} a\right) \cap F_{\mathfrak{p}}=\mathfrak{g}_{\mathfrak{p}} \beta . \quad$ Let $\left\{x_{i}, y_{i}, z\right\}$ be a canonical base of $V_{p}$ satisfying (4). Then the lattice $L$ defined by (3) is normal, maximal and $N(L)=\mathfrak{D}_{\mathfrak{p}} a$ or $\mathrm{D}_{\mathfrak{p}} \beta$ according as $n>1$ or $n=1$.

Proposition 3.6. Let $L$ be a $\mathfrak{g}_{\mathfrak{p}}$-lattice in $V_{\mathfrak{p}}$. Let $\mathfrak{b}$ be an $\mathfrak{o}_{\mathfrak{p}}$-ideal such that $N(L) \subset \mathfrak{b}$. Then there exists a maximal lattice $M$ such that $M \supset L, N(M)=\mathfrak{b}$ or $N(M)=\mathfrak{p}_{\mathfrak{p}} \cdot\left(\mathfrak{b} \cap F_{\mathfrak{p}}\right)$ according as $n>1$ or $n=1$.

We first show that, if Proposition 3.5 is true for $n$, then Proposition 3.6 is true for $n$. Let the notation be as in Proposition 3.6. By Proposition 2.8, we may assume that $L$ is maximal. Put $N(L)=\mathfrak{p}_{p} a, N(L) \cap F_{\mathfrak{p}}=\mathfrak{g}_{\mathfrak{p}} \beta$ with $a \in D_{\mathfrak{p}}$, $\beta \in F_{\mathrm{p}}$. If $n=1$, we put $a=\beta$. By Proposition 3.5, there exists a canonical base $\left\{x_{i}, y_{i}, z\right\}$ of $V_{\mathfrak{p}}$ satisfying (3) and (4). Put $\mathfrak{b}=\mathfrak{p}_{\mathfrak{p}} b, \mathfrak{b} \cap F_{\mathfrak{p}}=\mathfrak{g}_{\mathfrak{p} \gamma}, a=c b$, $\beta=\varepsilon \gamma$. Then $c \in \mathfrak{D}_{\mathfrak{p}}, \varepsilon \in \mathfrak{g}_{\mathfrak{p}}$. By Proposition 3.3, we can find an element $e$ of $\mathfrak{o}_{\mathfrak{p}}$ such that $N(e)=\varepsilon . \quad$ Put $M=\sum_{i=1}^{m} \mathfrak{b}_{\mathfrak{p}} c^{-1} x_{i}+\sum_{i=1}^{m} \mathfrak{o}_{\mathfrak{p}} y_{i}+\mathfrak{o}_{\mathfrak{p}} e^{-1} z . \quad$ As $f\left(c^{-1} x_{i}, y_{j}\right)=b \delta_{i j}$ and $f\left(e^{-1} z, e^{-1} z\right)=\gamma$, we see, from Proposition 3.5, that $M$ is a maximal lattice and $N(M)=\mathfrak{b}$ or $\mathfrak{b}_{\mathfrak{p}} \cdot\left(\mathfrak{b} \cap F_{\mathfrak{p}}\right)$ according as $n>1$ or $n=1$. By our construction of $M$, we have $M \supset L$. This proves Proposition 3.6.

Now we want to prove the converse part of Proposition 3.5. Define $L$ as 
in Proposition 3.5. It is clear that $L$ has $\mathfrak{p}_{\mathfrak{p}}$ as its order and $N(L)=\mathfrak{p}_{\mathfrak{p}} a$ or $\mathfrak{p}_{\mathfrak{p}} \beta$ according as $n>1$ or $n=1$. Let $M$ be a lattice with order $\mathfrak{p}_{\mathfrak{p}}$ such that $M \supset L$, $N(M)=N(L)$. Let $u=\sum_{i=1}^{m}\left(c_{i} x_{i}+d_{i} y_{i}\right)+e z$, with $c_{i}, d_{i}, e \in D_{\mathfrak{p}}$, be an element of $M$, the term $e z$ occurring only when $n$ is odd. As the $x_{i}$ and $y_{i}$ are contained in $M$, we have

$$
d_{i} a^{\prime}=f\left(u, x_{i}\right) \in N(M)=\mathfrak{p}_{\mathfrak{p}} a, \quad a c_{i}=f\left(u, y_{i}\right) \in N(M)=\mathfrak{p}_{p} a .
$$

This implies $d_{i} \in \mathfrak{D}_{\mathfrak{p}}, c_{i} \in \mathfrak{b}_{\mathfrak{p}}$. It follows that $e z=u-\sum_{i=1}^{m}\left(c_{i} x_{i}+d_{i} y_{i}\right) \in M$. Hence we have $N(e) \beta=f(e z, e z) \in N(M) \cap F_{\mathfrak{p}}=\mathfrak{g}_{\mathfrak{p}} \beta$, so that $N(e) \in \mathfrak{g}_{\mathfrak{p}}$, and hence $e \in \mathfrak{p}_{\mathrm{p}}$. Therefore $u$ must be contained in $L$; so we have $M=L$; this proves the maximality of $L$.

Let us prove the direct part of Proposition 3.5 by induction on $n$. If $n=1$, take a base $x$ of $V_{\mathfrak{p}}$ over $D_{\mathfrak{p}}$. Then $L$ is written in the form $L=\mathfrak{a} x$ with an $\mathfrak{o}_{\mathfrak{p}}$-ideal $\mathfrak{a}$. We have $N(L)=\mathfrak{o}_{\mathfrak{p}} N(\mathfrak{a}) f(x, x)$, so that $N(\mathfrak{a}) f(x, x)=\mathfrak{g}_{\mathfrak{p}} \beta$. By Proposition 3.3, there exists an element $b$ of $D_{p}$ such that $N(b)=\beta f(x, x)^{-1}$. Put $z=b x$. Then $f(z, z)=\beta, N(b) \mathfrak{y}_{\mathfrak{p}}=N(\mathfrak{a})$ and hence $\mathfrak{p}_{\mathfrak{p}} b=\mathfrak{a}$. We have therefore $L=\mathfrak{p}_{\sharp} z$. This proves the case $n=1$. Now suppose that $n>1$. By the remark after Proposition 3.4, $V_{\mathfrak{p}}$ contains an element $x \neq 0$ such that $f(x, x)=0$. Put $\mathfrak{c}=\left\{c \in D_{\mathfrak{p}} \mid c x \in L\right\}$. Obviously, $\mathfrak{c}$ is an $\mathfrak{o}_{\mathfrak{p}}$-ideal, so it is written in the form $\mathfrak{c}=\mathfrak{b}_{p} c_{0}$. Put $c_{0} x=x_{1}$. Then we see that

$$
\mathfrak{o}_{\mathfrak{p}}=\left\{c \in D_{\mathfrak{p}} \mid c x_{1} \in L\right\}
$$

and $f\left(x_{1}, x_{1}\right)=0 . \quad$ Put $\mathfrak{b}=f\left(x_{1}, L\right)$. It is clear that $\mathfrak{b}$ is an $\mathfrak{p}_{p}$-ideal. If $b \in N(L) \mathfrak{b}^{-1}$ and $u \in L$, we have $f\left(b x_{1}, u\right)=b f\left(x_{1}, u\right) \in \mathfrak{b} N(L) \mathfrak{b}^{-1}=N(L)$. Therefore, if $b, c$ $\in N(L) \mathfrak{b}^{-1}$ and $u, v \in L$, we have

$$
f\left(b x_{1}+u, c x_{1}+v\right)=f\left(b x_{1}, v\right)+f\left(u, c x_{1}\right)+f(u, v) \in N(L) .
$$

Put $M=N(L)^{-1} x_{1}+L$. The relation (6) shows that $N(M)=N(L)$. As $L$ is maximal, we must have $L=M$, so that $N(L) \mathfrak{b}^{-1} x_{1} \subset L$. By (5), we have $N(L) \mathfrak{b}^{-1} \subset \mathfrak{b}_{\mathfrak{p}}$, so that $N(L) \subset \mathfrak{b}$. As $\mathfrak{b}=f\left(x_{1}, L\right) \subset N(L)$, we must have $\mathfrak{b}=N(L)$ $=\mathfrak{o}_{p} a$. Hence there exists an element $y$ of $L$ such that $f\left(x_{1}, y\right)=a$. By Proposition 3.1, we have $\operatorname{Tr}\left(\mathfrak{b}_{\mathfrak{p}} a\right)=N(L) \cap F_{\mathfrak{p}} \ni-f(y, y)$. Therefore we can find an element $t$ of $\mathfrak{o}_{p}$ such that $\operatorname{Tr}(t a)=-f(y, y)$. Put $y_{1}=t x_{1}+y$. Then $y_{1} \in L$, and we have $f\left(x_{1}, y\right)=a, f\left(y_{1}, y_{1}\right)=0$. Put

$$
\begin{aligned}
& U=\left\{u \in V_{\mathfrak{p}} \mid f\left(x_{1}, u\right)=f\left(y_{1}, u\right)=0\right\}, \\
& K=U \cap L .
\end{aligned}
$$

For every $w \in V$, if we put $a^{-1} f\left(x_{1}, w\right)=d$ and $f\left(w, y_{1}\right) a^{-1}=c$, we see easily $w-c x_{1}-d^{\prime} y_{1} \in U$. This implies $V_{p}=D_{\mathfrak{p}} x_{1}+D_{\mathfrak{p}} y_{1}+U$. If $w \in L$, then $f\left(x_{1}, w\right)$ and $f\left(w, y_{1}\right)$ are contained in $N(L)=\mathfrak{p}_{\mathfrak{p}} a$, so that $c$ and $d$ are contained in $\mathfrak{o}_{\mathfrak{p}}$. 
We have therefore, $L=\mathfrak{o}_{\mathfrak{p}} x_{1}+\mathfrak{o}_{\mathfrak{p}} y_{1}+K$. Obviously, $K$ is a lattice in $U$ with the order $\mathfrak{o}_{\mathfrak{p}}$, and $N(K) \subset N(L)$. As $L$ is maximal, $K$ must be maximal; so we can apply our induction to $K$. By the assumption of induction, Proposition 3.6 is true for $n-2$. Therefore, if $n>3$, we must have $N(K)=N(L)=\mathfrak{p}_{p} a$, while if $n=3, N(K)=\mathfrak{d}_{\mathfrak{p}} \beta$. This completes our proof.

We call the base $\left\{x_{i}, y_{i}, z\right\}$ in the above proposition a canonical base of $L$.

Proposition 3.7. Let $L$ and $M$ be maximal lattices in $V_{p}$. If $L \sigma=M$ for an element $\sigma$ of $G\left(V_{\mathfrak{p}}, f\right)$, then $N(L)^{-1} N(M)$ is an even power of the maximal ideal of $\mathfrak{o}_{\mathfrak{p}}$, namely $N(L)^{-1} N(M)=\mathfrak{o}_{\mathfrak{p}} \alpha$ for an element $\alpha$ of $F_{\mathfrak{p}}$. Conversely, if $\alpha N(L)=N(M)$ with $\alpha \in F$, there exists an element $\sigma$ of $G\left(V_{\mathfrak{p}}, f\right)$ such that $L \sigma$ $=M, N(\sigma)=\alpha$.

Proof. If $L \sigma=M$ for some $\sigma \in G\left(V_{p}, f\right)$, we have $N(L) N(\sigma)=N(M)$, so that $N(L)^{-1} N(M)=N(\sigma) \mathfrak{o}_{\mathfrak{p}}$. This proves the first assertion. Conversely, suppose that $\alpha N(L)=N(M)$ with $\alpha \in F_{\mathrm{p}}$. Put $N(L)=\mathfrak{p}_{p} a, N(L) \cap F_{\mathrm{p}}=\mathrm{g}_{\mathrm{p}} \beta$ with $a \in D_{\mathfrak{p}}, \beta \in F_{\mathfrak{p}}$. Then we have $N(M)=\mathfrak{o}_{\mathfrak{p}} \alpha a, N(M) \cap F_{\mathfrak{p}}=\mathfrak{g}_{\mathfrak{p}} \alpha \beta$. By Proposition 3.5, there exists a canonical base $\left\{x_{i}, y_{i}, z\right\}$ of $L$ such that $f\left(x_{i}, y_{j}\right)=a \delta_{i j}$, $f(z, z)=\beta$, and a canonical base $\left\{u_{i}, v_{i}, w\right\}$ of $M$ such that $f\left(u_{i}, v_{j}\right)=\alpha \alpha \delta_{i j}$, $f(w, w)=\alpha \beta$. Define an element $\sigma$ of $E\left(V_{\mathfrak{p}}, D_{\mathfrak{p}}\right)$ by $x_{i} \sigma=u_{i}, y_{i} \sigma=v_{i}, z \sigma=w$. Then we see easily that $\sigma \in G\left(V_{p}, f\right), L \sigma=M$ and $N(\sigma)=\alpha$. This completes our proof.

Proposition 3.8. Let $L$ be a maximal lattice in $V_{\mathfrak{p}}$. If $n>1$, there exists a base $\left\{u_{1}, \cdots, u_{n}\right\}$ of $V_{p}$ over $D_{p}$ such that $L=\mathfrak{o}_{p} u_{1}+\cdots+\mathfrak{p}_{p} u_{n}, f\left(u_{i}, u_{i}\right)=0$ for $1 \leqq i \leqq n$.

Proof. Take a canonical base $\left\{x_{i}, y_{i}, z\right\}$ of $L$. If $n$ is even, our assertion is a consequence of the relation $f\left(x_{i}, x_{i}\right)=f\left(y_{i}, y_{i}\right)=0$. Suppose that $n$ is odd. The elements $a$ and $\beta$ being as in Proposition 3.5, we get, by Proposition 3.1, $\beta \in F_{\mathfrak{p}} \cap \mathfrak{o}_{\mathfrak{p}} a=\operatorname{Tr}\left(\mathfrak{o}_{\mathfrak{p}} a\right)$. Hence there exists an element $b$ of $\mathfrak{o}_{\mathfrak{p}}$ such that $\operatorname{Tr}(b a)=\beta$. Put $w=z+b x_{1}-y_{1}$. Then we have $f(w, w)=0$ and $L=\mathfrak{p}_{\mathrm{p}} x_{1}$ $+\mathfrak{p}_{\mathfrak{p}} y_{1}+\cdots+\mathfrak{p}_{\mathfrak{p}} x_{m}+\mathfrak{p}_{\mathfrak{p}} y_{m}+\mathfrak{p}_{\mathfrak{p}} w$. This proves our proposition.

Proposition 3.9. Let $L$ and $M$ be maximal lattices in $V_{p}$. Put $N(L)=h \mathfrak{d}_{\mathrm{p}}$, $N(L) \cap F_{\mathfrak{p}}=\eta g_{\mathfrak{p}}$ with $h \in D_{\mathfrak{p}}, \eta \in F_{\mathfrak{p}}$, and suppose that $N(M)=\alpha N(L)$ for an element $\alpha$ of $F_{\mathfrak{p}}$. Then there exist a canonical base $\left\{x_{i}, y_{i}, z\right\}$ of $V_{\mathfrak{p}}$ and elements $a_{i}, b_{i}, c$ of $D_{p}$ such that

$$
\begin{aligned}
& L=\mathfrak{p}_{\mathfrak{p}} x_{1}+\mathfrak{p}_{\mathfrak{p}} y_{1}+\cdots+\mathfrak{p}_{\mathfrak{p}} x_{m}+\mathfrak{p}_{\mathfrak{p}} y_{m}+\mathfrak{p}_{\mathfrak{p}} z, \\
& M=\mathfrak{o}_{\mathfrak{p}} a_{1} x_{1}+\mathfrak{o}_{\mathfrak{p}} b_{1} y_{1}+\cdots+\mathfrak{o}_{\mathfrak{p}} a_{m} x_{m}+\mathfrak{o}_{\mathfrak{p}} b_{m} y_{m}+\mathfrak{o}_{\mathfrak{p}} c z \text {, } \\
& f\left(x_{i}, y_{j}\right)=h \delta_{i j}, \quad f(z, z)=\eta, \\
& a_{1} h b_{1}^{\prime}=\cdots=a_{m} h b_{m}^{\prime}=\alpha h, \quad c c^{\prime}=\alpha, \\
& \mathfrak{o}_{\mathfrak{p}} a_{1} \supset \cdots \supset \mathfrak{p}_{\mathfrak{p}} a_{m} \supset \mathfrak{p}_{\mathfrak{p}} c \supset \mathfrak{p}_{\mathfrak{p}} b_{m} \supset \cdots \supset \mathfrak{p}_{\mathfrak{p}} b_{1} \text {, }
\end{aligned}
$$

where $z$ and $c$ occur only when $n$ is odd. 
ProOF. We proceed by induction on $n$. If $n=1$, this is obvious. Suppose that $n>1$. Put $\mathfrak{e}=\left\{e \in D_{\mathfrak{p}} \mid e M \subset L\right\}$. As $\mathfrak{e}$ is an $\mathfrak{o}_{\mathfrak{p}}$-ideal, we have $\mathfrak{e}=\mathfrak{D}_{\mathfrak{p}} e_{0}$. for an element $e_{0} \in \mathfrak{D}_{\mathfrak{p}}$. Put $e_{0} M=M_{1}$; then $N\left(M_{1}\right)=N\left(e_{0}\right) N(L)$. If we prove our proposition for $M_{1}$, we get easily the assertion for $M$. In fact, suppose that we get a canonical base $\left\{x_{i}, y_{i}, z\right\}$ of $V_{p}$ and elements $r_{i}, s_{i}, t$ of $D_{p}$ such that $L=\sum_{i=1}^{m}\left(\mathfrak{p}_{\mathrm{p}} x_{i}+\mathfrak{o}_{\mathfrak{p}} y_{i}\right)+\mathfrak{o}_{\mathfrak{p}} z, \quad M_{1}=\sum_{i=1}^{m}\left(\mathfrak{o}_{\mathfrak{p}} r_{i} x_{i}+\mathfrak{o}_{\mathfrak{p}} s_{i} y_{i}\right)+\mathfrak{o}_{\mathrm{p}} t z, \quad f\left(x_{i}, y_{i}\right)=h, f(z, z)=\eta, \quad r_{i} h s_{i}^{\prime}$ $=N\left(e_{0}\right) \alpha h, t t^{\prime}=N\left(e_{0}\right) \alpha, \mathfrak{D}_{\mathfrak{p}} r_{1} \supset \cdots \supset \mathfrak{D}_{\mathfrak{p}} r_{m} \supset \mathfrak{D}_{\mathfrak{p}} t \supset \mathfrak{D}_{\mathfrak{p}} s_{m} \supset \cdots \supset \mathfrak{D}_{\mathfrak{p}} s_{1} . \quad$ Put $a_{i}=e_{0}^{-1} r_{i}, b_{i}$ $=h^{-1} e_{0}^{-1} h s_{i}, c=e_{0}^{-1} t$. Then we can easily verify that $\left\{x_{i}, y_{i}, z\right\}$ and $\left\{a_{i}, b_{i}, c\right\}$ have the properties of our proposition for $M$ and $L$. Therefore we may assume that $M=M_{1}$, namely $\mathfrak{D}_{\mathfrak{p}}=\left\{e \in D_{\mathfrak{p}} \mid e M \subset L\right\}$. Let $\Pi$ be a prime element of $\mathfrak{p}_{\mathfrak{p}}$. By Proposition 3.8, $M$ contains an element $x_{1}$ such that $f\left(x_{1}, x_{1}\right)=0, \Pi^{-1} x_{1} \notin L$. Namely, the relation (5) holds for this $\left\{x_{1}, L\right\}$. Hence, applying the proof of Proposition 3.5 to the present case, we get an element $y_{1}$ of $L$ such that $f\left(x_{1}, y_{1}\right)=h, f\left(y_{1}, y_{1}\right)=0$. By Proposition 2.7, we have $\alpha L \subset M$, so that $\alpha y_{1} \in M$. Put $U=\left\{u \in V_{\mathfrak{p}} \mid f\left(x_{1}, u\right)=f\left(y_{1}, u\right)=0\right\}, L_{0}=U \cap L, M_{0}=U \cap M$. Then, as in the proof of Proposition 3.5, we obtain

$$
V_{\mathfrak{p}}=D_{\mathfrak{p}} x_{1}+D_{\mathfrak{p}} y_{1}+U, \quad L=\mathfrak{o}_{\mathfrak{p}} x_{1}+\mathfrak{o}_{\mathfrak{p}} y_{1}+L_{0} ;
$$

and $L_{0}$ is a maximal lattice in $U$ such that $N\left(L_{0}\right)=h 0_{p}$ or $\eta 0_{p}$ according as $n>1$ or $n=1$. Let $w=d x_{1}+e y_{1}+w_{0}$, with $d \in \mathrm{D}_{\mathfrak{p}}, e \in \mathrm{D}_{\mathrm{p}}, w_{0} \in L_{0}$, be an element of $M$. Then we have $e=f\left(w, x_{1}\right) \in N(M)=\mathfrak{D}_{p} \alpha h$, so that $e \in \mathfrak{D}_{\mathfrak{p}} \alpha, e y_{1} \in \mathfrak{D}_{p} \alpha y_{1} \subset M$, and hence $w_{0}=w-d x_{1}-e y_{1} \in M \cap U=M_{0}$. This implies $M=\mathfrak{p}_{p} x_{1}+\mathfrak{o}_{p} \alpha y_{1}+M_{0}$. We observe that $M_{0}$ is a maximal lattice in $U$ such that $N\left(M_{0}\right)=\alpha N\left(L_{0}\right)$. Therefore we can apply our induction to $L_{0}$ and $M_{0}$. Then we obtain a canonical base $\left\{x_{2}, \cdots, x_{m}, y_{2}, \cdots, y_{m}, z\right\}$ of $U$ and elements $a_{2}, \cdots, a_{m}, b_{2}, \cdots, b_{m}, c$ of $D_{\mathfrak{p}}$ such that $L_{0}=\sum_{i=2}^{m}\left(\mathfrak{o}_{\mathfrak{p}} x_{i}+\mathfrak{o}_{\mathfrak{p}} y_{i}\right)+\mathfrak{o}_{\mathfrak{p}} z, M_{0}=\sum_{i=2}^{m}\left(\mathfrak{p}_{\mathfrak{p}} a_{i} x_{i}+\mathfrak{o}_{\mathfrak{p}} b_{i} y_{i}\right)+\mathfrak{o}_{\mathfrak{p}} c z, f\left(x_{i}, y_{i}\right)=h$ for $2 \leqq i \leqq m, f(z, z)=\eta, a_{2} h b_{2}^{\prime}=\cdots=a_{m} h b_{m}^{\prime}=\alpha h, c c^{\prime}=\alpha, \mathfrak{p}_{p} a_{2} \supset \cdots \supset \mathfrak{D}_{\mathfrak{p}} a_{m} \supset \mathfrak{D}_{p} c \supset \mathfrak{D}_{p} b_{m}$ $\supset \cdots \supset \mathfrak{o}_{p} b_{2}$. As $L_{0} \supset M_{0}$, we have $\mathfrak{o}_{\mathfrak{p}} \ni a_{2}$, so that $\mathfrak{o}_{p} b_{2} \supset \mathfrak{o}_{p} \alpha$. Putting $a_{1}=1$ and $b_{1}=\alpha$, we obtain our assertion for $L$ and $M$. This completes the proof.

Proposition 3.10. Let $L$ be a maximal lattice in $V_{\mathrm{p}}$. Put $N(L)=h_{\mathfrak{p}_{\mathrm{p}}}$, $N(L) \cap F_{\mathfrak{p}}=\eta g_{\mathfrak{p}}$ with $h \in D_{\mathfrak{p}}, \eta \in F_{\mathfrak{p}} . \quad$ Let $\left\{u_{i}, v_{i}, w\right\}$ be a canonical base of $L$ such that $f\left(u_{i}, v_{j}\right)=h \delta_{i j}, f(w, w)=\eta$. Denote by $\Gamma^{0}$ the subgroup of $G^{0}\left(V_{\mathfrak{p}}, f\right)$ consisting of the elements $r \in G^{0}\left(V_{p}, f\right)$ such that $L_{r}=L$, and by $\Delta$ the set of elements $\sigma$ of $G\left(V_{\mathfrak{p}}, f\right)$ such that $u_{i} \sigma=a_{i} u_{i}, v_{i} \sigma=b_{i} v_{i}, w \sigma=c w$ with elements $a_{i}, b_{i}, c$ of $D_{\mathfrak{p}}$ satisfying the relation

$$
\mathfrak{p}_{p} a_{1} \supset \cdots \supset \mathfrak{p}_{p} a_{m} \supset \mathfrak{p}_{p} c \supset \mathfrak{p}_{p} b_{m} \supset \cdots \supset \mathfrak{o}_{p} b_{1} .
$$

Then we have $G\left(V_{\mathrm{p}}, f\right)=\Gamma^{0} \cdot \Delta \cdot \Gamma^{0}$.

Proof. Let $\tau$ be an element of $G\left(V_{\sharp}, f\right)$. Put $M=L \tau, \alpha=N(\tau)$, and apply Proposition 3.9 to this $\{L, M, \alpha\}$. Then we get a canonical base $\left\{x_{i}, y_{i}, z\right\}$ of 
$L$ and elements $a_{i}, b_{i}, c$ of $D_{p}$ with the properties of that proposition. Define two elements $\gamma$ and $\sigma$ of $E\left(V_{\mathfrak{p}}, A_{\mathfrak{p}}\right)$ by $u_{i} \gamma=x_{i}, v_{i} \gamma=y_{i}, w \gamma=z, u_{i} \sigma=a_{i} u_{i}, v_{i} \sigma$ $=b_{i} v_{i}, w \sigma=c w$. We see easily that $r \in \Gamma^{0}$ and $\sigma \in \Delta, N(\sigma)=\alpha$. Further we have $L \sigma \gamma=L \tau$. Hence if we put $\varepsilon \sigma \gamma=\tau$, we have $L \varepsilon=L, \varepsilon \in G\left(V_{\mathfrak{p}}, f\right), N(\varepsilon)$ $=1$, so that $\varepsilon \in \Gamma^{0}$. It follows that $\tau=\varepsilon \sigma \gamma \in \Gamma^{0} \cdot \Delta \cdot \Gamma^{0}$. Our proposition is thereby proved.

Notation being as in Proposition 3.9, we call $\left\{\mathrm{p}_{p} a_{1}, \cdots, \mathrm{D}_{\mathrm{p}} a_{m}, \mathrm{D}_{\mathrm{p}} c, \mathrm{D}_{\mathrm{p}} b_{1}, \cdots, \mathrm{D}_{\mathrm{p}} b_{m}\right\}$ the set of elementary divisors of $M$ relative to $L$ and denote it by $\{L: M\}$.

Proposition 3.11. Let $L, M, K$ be maximal lattices in $V_{\mathfrak{p}}$ such that $N(M)$ $=\alpha N(L), N(K)=\beta N(L)$ with $\alpha, \beta \in F_{\mathrm{p}}$. Then, there exists an element $\sigma$ of $G^{0}\left(V_{p}, f\right)$ such that $L \sigma=L$ and $M \sigma=K$, if and only if $\{L: M\}=\{L: K\}$.

By virtue of Proposition 3.9, this can be proved by the same argument as in the proof of Proposition 2.13. When $L \supset M$ and $L \supset K$, the equality $\{L: M\}=\{L: K\}$ holds if and only if $L / M$ and $L / K$ are isomorphic as $\mathfrak{D}_{p^{-}}$ modules.

3.3. Local approximation theorem. Let $A_{p}$ be a quaternion algebra over $F_{\mathfrak{p}}$, which may be or may not be a division algebra. Let $\mathfrak{o}_{\mathfrak{p}}$ be a maximal order in $A_{\text {p. }}$.

Proposition 3.12. Let $V_{\mathfrak{p}}$ be an $A_{p}$-space of dimension $n$ and $f$ be a nondegenerate $Q$-hermitian form on $V_{\mathrm{p}}$. Let $L$ be a maximal lattice in $V_{p}$ such that $N(L)=\mathfrak{p}_{\mathfrak{p}}$. Then there exists a base $\left\{x_{1}, \cdots, x_{n}\right\}$ of $V_{\mathfrak{p}}$ over $A_{\mathfrak{p}}$ such that $f\left(x_{i}, x_{j}\right)=\delta_{i j}$ and $L=\mathfrak{p}_{p} x_{1}+\cdots+\mathfrak{o}_{p} x_{n}$.

Proof. By Proposition 2.1 and Proposition 3.3, $V_{\mathfrak{p}}$ has a base $\left\{y_{1}, \cdots, y_{n}\right\}$ over $A_{\mathfrak{p}}$ such that $f\left(y_{i}, y_{j}\right)=\delta_{i j}$. Put $M=\mathfrak{o}_{\mathfrak{p}} y_{1}+\cdots+\mathfrak{o}_{\mathfrak{p}} y_{n}$. By Proposition 2.6, $M$ is a maximal lattice in $V_{\mathfrak{p}}$ and $N(M)=\mathfrak{o}_{\mathfrak{p}}$. By Proposition 2.11 (if $A_{\mathfrak{p}}=M_{2}\left(F_{\mathfrak{p}}\right)$ ) and by Proposition 3.7 (if $A_{\mathfrak{p}}=D_{\mathfrak{p}}$ ), there exists an element $\sigma$ of $G^{0}\left(V_{\mathfrak{p}}, f\right.$ ) such that $L=M \sigma$. Putting $x_{i}=y_{i} \sigma$ for $1 \leqq i \leqq n$, we get the desired result.

Proposition 3.13. Let $V_{\mathfrak{p}}$ and $U_{\mathfrak{p}}$ be $A_{p}$-spaces of the same dimension; let $f$ and $h$ be non-degenerate $Q$-hermitian forms on $V_{\mathfrak{p}}$ and on $U_{\mathfrak{p}}$, respectively. Let $L$ and $M$ be maximal lattices in $V_{\mathfrak{p}}$ and in $U_{\mathfrak{p}}$, respectively, such that $N_{f}(L)$ $=N_{h}(M)=\mathrm{D}_{\mathrm{p}}$. Let $\tau$ be an $A_{\mathrm{p}}$-linear mapping of $V_{\mathrm{p}}$ into $U_{\mathrm{p}}$ such that $L \tau \subset M$, $f(x, y) \equiv h(x \tau, y \tau) \bmod \mathfrak{p}^{\lambda_{\mathfrak{p}}}$ for every $x, y \in L$, where $\lambda$ is an integer $\geqq 0$. Then there exists an $A_{p}$-isomorphism $\sigma$ of $V_{p}$ onto $U_{\downarrow}$ such that $L \sigma=M, f(x, y)=h(x \sigma, y \sigma)$ for every $x, y \in V_{p}$ and $L(\sigma-\tau) \subset p^{\lambda} M$.

Proof. Our proposition is clear if $\lambda=0$; so we assume $\lambda \geqq 1$. Let $n$ be the common dimension of $V_{p}$ and $U_{p}$. We proceed by induction on $n$. By Proposition 3.12, there exists a base $\left\{u_{1}, \cdots, u_{n}\right\}$ of $U_{p}$ over $A_{\mathfrak{p}}$ such that $M$ $=\mathfrak{o}_{p} u_{1}+\cdots+\mathfrak{o}_{\mathfrak{p}} u_{n}, h\left(u_{i}, u_{j}\right)=\delta_{i j}$; and $L$ contains an element $v$ such that $f(v, v)=1$. Put $v \tau=\sum_{i=1}^{n} \alpha_{i} u_{i}$ with $a_{i} \in \mathfrak{D}_{\mathfrak{p}} . \quad$ Then $1=f(v, v) \equiv h(v \tau, v \tau)=\sum_{i=1}^{n} N\left(a_{i}\right) \bmod \mathfrak{p}^{\lambda} \mathfrak{D}_{\mathfrak{p}}$. 
Therefore $N\left(a_{i}\right)$ is a unit of $\mathfrak{g}_{\mathfrak{p}}$ for some $i$, say 1 . Put $\beta=1-\sum_{i=2}^{n} N\left(a_{i}\right)$. Then $N\left(a_{1}\right) \equiv \beta \bmod \mathfrak{p}^{\lambda_{\mathfrak{p}}}$, and hence $\beta$ is a unit of $\mathfrak{g}_{\mathfrak{p}}$. By Proposition 3.2, there exists an element $b$ of $\mathfrak{D}_{\mathfrak{p}}$ such that $b \equiv a_{1} \bmod \mathfrak{p}^{\lambda_{\mathfrak{p}}}$ and $N(b)=\beta$. Put $w$ $=b u_{1}+\sum_{i=2}^{n} a_{i} u_{i}$. Then $h(w, w)=1, w \equiv v \tau \bmod \mathfrak{p}^{\lambda} M$, and $w \in M$. Put

$$
\begin{aligned}
V^{0}=\left\{x \in V_{\mathfrak{p}} \mid f(x, v)=0\right\}, & U^{0}=\left\{x \in U_{\mathfrak{p}} \mid h(x, w)=0\right\}, \\
L^{0}=L \cap V^{0}, & M^{0}=M \cap U^{0} .
\end{aligned}
$$

As $f(v, v)=h(w, w)=1$, we obtain

$$
\begin{array}{ll}
V_{\mathfrak{p}}=A_{\mathfrak{p}} v+V^{0}, & U_{\mathfrak{p}}=A_{\mathfrak{p}} w+V^{0}, \\
L=\mathfrak{}_{\mathfrak{p}} v+L^{0}, & M=\mathfrak{p}_{\mathfrak{p}} w+M^{0} .
\end{array}
$$

It can be easily seen that $L$ and $M$ are respectively maximal lattices in $V^{0}$ and $U^{0}$; and $N_{f}\left(L^{0}\right)=N_{h}\left(M^{0}\right)=\mathfrak{p}_{\mathfrak{p}}$. Now define an $A_{p}$-linear mapping $\rho$ of $V^{0}$ into $U^{0}$ by $x \tau=t w+x \rho$ for $x \in V^{0}$, where $t \in A_{p}$. We see easily $L^{0} \rho \subset M^{0}$. If $x \in L^{0}$ and $x \tau=t w+x \rho$, we have $0=f(v, x) \equiv h(v \tau, x \tau) \equiv h(w, t w+x \rho)=t \bmod p^{\lambda_{0}}$. This shows $x \tau \equiv x \rho \bmod \mathfrak{p}^{\lambda} M$ for $x \in L^{0}$. If further $y \in L^{0}$, we have $f(x, y)$ $\equiv h(x \tau, y \tau) \equiv h(x \rho, y \rho) \bmod \mathfrak{p}^{\lambda} \mathfrak{o}_{\mathfrak{p}}$. Therefore we can apply induction to $L^{0}, M^{0}, \rho$. Namely there exists an $A_{p}$-isomorphism $\sigma^{0}$ of $V^{0}$ onto $U^{0}$ such that $L^{0} \sigma^{0}=M^{0}$, $f(x, y)=h\left(x \sigma^{0}, y \sigma^{0}\right)$ for every $x, y \in V^{0}$, and $L^{0}\left(\sigma^{0}-\rho\right) \subset \mathfrak{p}^{\lambda} M^{0}$. Now define an $A_{\mathfrak{p}}$-isomorphism $\sigma$ of $V_{\mathfrak{p}}$ onto $U_{\mathfrak{p}}$ by $v \sigma=w$ and $x \sigma=x \sigma^{0}$ for every $x \in V^{0}$. Then we have clearly $L \sigma=M, f(x, y)=h(x \sigma, y \sigma)$ for every $x, y \in V_{p}$. Furthermore, $v \sigma=w \equiv v \tau \bmod \mathfrak{p}^{\lambda} M$; and if $x \in L^{0}, x \sigma=x \sigma^{0} \equiv x \rho \equiv x \tau \bmod \mathfrak{p}^{\lambda} M$. Therefore $L(\sigma-\tau) \subset \mathfrak{p}^{\lambda} M$. This completes our proof.

\section{$\S 4$. Global theory of $Q$-hermitian forms.}

In this section, we always mean by $F$ an algebraic number field of finite degree, and by $g$ the ring of integers in $F$. For every prime ideal $\mathfrak{p}$ of $F, F_{\mathfrak{p}}$ and $\mathfrak{g}_{\mathfrak{p}}$ denote respectively the $\mathfrak{p}$-completions of $F$ and $\mathfrak{g}$. We denote by $\mathfrak{p}_{\varsigma_{\kappa}}$ for $1 \leqq \kappa \leqq v$ the infinite prime spots of $F$ and by $F_{\kappa}$ the completion of $F$ with respect to $\mathfrak{p}_{\infty}$.

4.1. Quaternion algebras over an algebraic number field. Let $A$ be a quaternion algebra over $F$. For each prime ideal $\mathfrak{p}$ of $F$, and for each infinite prime spot $\mathfrak{p}_{\infty \kappa}$ of $F$, we put

$$
A_{\mathfrak{p}}=A \underset{F}{\otimes} F_{\mathfrak{p}}, \quad A_{\kappa}=\underset{F}{A} \bigotimes_{\kappa} F_{\kappa} .
$$

$A$ finite or infinite prime spot of $F$ is called ramified in $A / F$ if the corresponding completion $A_{\mathfrak{p}}$ or $A_{\kappa}$ is a division algebra. Let $\mathrm{o}$ be a maximal order in $A$. Let $\mathscr{D}=\mathfrak{D}(\mathfrak{D} / \mathfrak{g})$ be the different of $\mathfrak{o}$ with respect to $\mathfrak{g}$. Then we have 
$\mathfrak{D}=\prod_{i=1}^{s} \mathfrak{Q}_{i}, \mathfrak{Q}_{i}^{2}=\mathfrak{q}_{i}$, where the $\mathfrak{q}_{i}$ are all the prime ideals of $F$ which are ramified in $A / F$, and $\mathfrak{Q}_{i}$ is a prime $\mathrm{p}$-ideal. Every two-sided $\mathrm{D}$-ideal $a$ is written in the form $\mathfrak{a}=\Pi \Omega_{i}^{e_{i}} \cdot \mathfrak{a}_{0}$, where $e_{i}=0$ or 1 , and $\mathfrak{a}_{0}$ is a $\mathfrak{g}$-ideal.

PROPOSITION 4.1. Let $\mathfrak{p}_{\infty_{1}}, \cdots, \mathfrak{p}_{\infty u}$ be the infinite prime spots of $F$ ramified in $A / F$, and $\xi$ be a non-zero element of $F$. Then there exists an element $x$ of $A$ such that $N(x)=\xi$, if and only if $\xi \equiv 1 \bmod \mathfrak{p}_{\infty} \cdots \mathfrak{p}_{\infty u}$.

Proof. Consider $N(x)=x x^{\prime}$ as a quadratic form on $A$ over $F$. By Hasse's theorem, the equation $x x^{\prime}=\xi$ has a solution if and only if it is solvable in every local fields. Our proposition is therefore an immediate consequence of Proposition 3.3.

We call $A$ definite (or totally definite) if all the infinite prime spots of $F$ are ramified in $A / F$, and call $A$ indefinite otherwise. If $A$ is definite, then $F$ must be totally real and $A_{\kappa}=\boldsymbol{K}$ for every infinite prime spot $\mathfrak{p}_{\propto_{\kappa}}$ of $F$. Now the following two fundamental lemmas are due to Eichler; they are originally given in a more general case (cf. [5, Satz 5]).

Lemma 4.2. Suppose that $A$ is indefinite. Let $\mathrm{D}$ be a maximal order in $A$ and let $\mathfrak{p}_{\infty 1}, \cdots, \mathfrak{p}_{\infty u}$ be the infinite prime spots ramified in $A / F$. Let $\mathfrak{b}$ and $\mathfrak{c}$ be left $\mathrm{D}$-ideals. Then there exists an element $x$ of $A$ such that $\mathfrak{b}=\mathfrak{c} x$, if and only if $N(\mathfrak{b})$ and $N(\mathfrak{c})$ belong to the same ideal-class modulo $\mathfrak{p}_{\infty_{1}} \cdots \mathfrak{p}_{\infty u}$ of $F$.

LEMMA 4.3. Notation and assumption being as in Lemma 4.2, let a be an integral two-sided $\mathfrak{D}$-ideal. Let $\beta$ be an element of $\mathrm{g}$ and $b$ an element of $\mathfrak{D}$ such that $\beta \equiv 1 \bmod \mathfrak{p}_{\infty 1} \cdots \mathfrak{p}_{\infty u}, N(b) \equiv \beta \bmod ^{*}(\mathfrak{a} \cap F)$. Then there exists an element $b_{0}$ of $\mathfrak{0}$ such that $b \equiv b_{0} \bmod \mathfrak{a}, N\left(b_{0}\right)=\beta$.

Here $\bmod *$ means the multiplicative congruence. Lemma 4.2 is easily derived from Lemma 4.3 (cf. [5, p. 239]). Our later discussion will prove this fact as a particular case.

4.2. Hasse principle for $\boldsymbol{Q}$-hermitian forms. In view of Proposition 3.3, there exists, among the quaternion algebras over local fields $F_{\mathfrak{p}}$ and $F_{\kappa}$, only one which does not satisfy the condition (D) of Proposition 2.1 ; it is the division ring $\boldsymbol{K}$ of real quaternions. Let $V$ be a $\boldsymbol{K}$-space of dimension $n$ and $f$ a non-degenerate $Q$-hermitian form on $V$. Then there exists a base $\left\{x_{1}, \cdots, x_{n}\right\}$ of $V$ over $\boldsymbol{K}$ such that $f\left(x_{i}, x_{j}\right)=\varepsilon_{i} \delta_{i j}$ for $1 \leqq i \leqq n, 1 \leqq j \leqq n$ and $\varepsilon_{i}=1$ for $1 \leqq i \leqq \nu, \varepsilon_{i}=-1$ for $\nu<i \leqq n$. The integer $\nu$ is uniquely determined by $f$. We put $\nu=\nu(f)$.

Let $A$ be a quaternion algebra over $F$ and let $\mathfrak{p}_{\infty 1}, \cdots, \mathfrak{p}_{\infty u}$ be all the infinite prime spots of $F$ ramified in $A / F$. Consider an $A$-space $V$ and a nondegenerate $Q$-hermitian form $f$ on $V$. Put $V_{\kappa}=V \otimes_{F} F_{\kappa}$ for $1 \leqq \kappa \leqq u$. Then $V_{\kappa}$ can be considered as an $A_{\kappa}$-space in a natural manner; and $f$ is uniquely extended to a non-degenerate $Q$-hermitian form $f_{\kappa}$ on $V_{\kappa}$. As $A_{\kappa}$ is isomorphic 
to $\boldsymbol{K}$, we can define $\nu\left(f_{k}\right)$. We put $\nu_{k}(f)=\nu\left(f_{\kappa}\right)$. Now, by Ramanathan [7], the structure of $\{V, f\}$ is completely determined by the $\nu_{k}(f)$. We state this result in the following form.

LEMMA 4.4. Let $f$ and $g$ be non-degenerate $Q$-hermitian forms on an $A$-space $V$. There exists an element $\sigma$ of $G L(V, A)$ such that $f(x \sigma, y \sigma)=g(x, y)$ for every $x, y$ of $V$, if and only if $\nu_{\kappa}(f)=\nu_{\kappa}(g)$ for every infinite prime spot $\mathfrak{p}_{\infty}$ of $F$ ramified in $A / F$.

This can be proved easily by means of Proposition 4.1 and the approximation theorem in the number field $F$.

4.3. Adele-group of $\boldsymbol{G}(\boldsymbol{V}, \boldsymbol{f})$. Let $A$ be a quaternion algebra over $F$ and $V$ an $A$-space of dimension $n$. For each prime ideal $\mathfrak{p}$ of $F$ and for each infinite prime spot $\mathfrak{p}_{\infty \kappa}$ of $F$, we put

$$
V_{p}=V \bigotimes_{F} F_{p}, \quad V_{\kappa}=V \bigotimes_{F} F_{\kappa} .
$$

Then $V_{\mathfrak{p}}$ (resp. $V_{k}$ ) can be considered as an $A_{\mathfrak{p}}$-space (resp. $A_{\kappa}$-space) in a natural manner. Let $f$ be a non-degenerate $Q$-hermitian form on $V$. We extend $f$ to non-degenerate $Q$-hermitian forms on $V_{\mathfrak{p}}$ and on $V_{k}$, and denote them again by $f$. Put now $G=G(V, f), G_{\mathfrak{p}}=G\left(V_{\mathfrak{p}}, f\right), G_{\kappa}=G\left(V_{\kappa}, f\right)$. Then $G_{\mathfrak{p}}, G_{k}$ are locally compact topological groups with usual topology. Let $L$ be a $g$ lattice in $V$. For each $\mathfrak{p}$, denote by $\mathfrak{H}_{\mathfrak{p}}$ the set of elements $\tau$ of $G_{\mathfrak{p}}$ such that $L_{p} \tau=L_{p}$. Then $\mathfrak{U}_{p}$ is a compact subgroup of $G_{p}$. Put

$$
\mathfrak{H}_{L}=\prod_{p} \mathfrak{H}_{\mathfrak{p}} \times \prod_{\kappa} G_{\kappa} .
$$

By the product topology, $\mathfrak{u}_{L}$ is a locally compact group. Now we define the adele-group $\&$ of $G(V, f)$ as the set of elements $\left(\sigma_{\mathfrak{p}}, \sigma_{\kappa}\right)$ of $\prod_{\mathfrak{p}} G_{\mathfrak{p}} \times \prod_{\kappa} G_{\kappa}$ such that $\sigma_{\mathfrak{p}} \in \mathfrak{H}_{\mathfrak{p}}$ for all except a finite number of $\mathfrak{p}$. Define a topology of $\mathbb{S}$ so that $\mathfrak{H}_{L}$ is an open subgroup of $\mathbb{S}$. Then $\mathbb{Q}$ becomes a locally compact group. The topological group $\mathbb{B}$ is determined independently of the choice of $L$. By the injection $\sigma \rightarrow(\cdots, \sigma, \sigma, \cdots), G$ can be considered as a discrete subgroup of $\mathbb{B}$. By a general theorem of Borel [1], $\&$ is the union of a finite number of double cosets $\mathfrak{H}_{L} \xi G$ with $\xi \in \mathbb{S}$ (cf. also Weil $[\mathbf{1 1}, \mathbf{1 2}]$ ).

4.4. Classes and Genera of maximal lattices. Notation being as in $\S 4.3$, let $\mathfrak{D}$ be a maximal order in $A$, and $\mathfrak{D}_{\mathfrak{p}}=\mathfrak{g}_{\mathfrak{p}} \mathfrak{D}$. We denote by $\mathfrak{L}(\mathfrak{D})$ the set of all maximal lattices in $V$ with the order $\mathfrak{o}$. Let $L$ and $M$ be two members of $2(\mathfrak{D})$. We say that $L$ and $M$ belong to the same genus, if there exists, for each prime ideal $\mathfrak{p}$ of $F$, an element $\sigma_{\mathfrak{p}}$ of $G\left(V_{\mathfrak{p}}, f\right)$ such that $L_{p} \sigma_{\mathfrak{p}}=M_{\mathfrak{p}}$. Further we say that $L$ and $M$ belong to the same class, if there exists an element $\sigma$ of $G(V, f)$ such that $L \sigma=M$.

Proposition 4.5. If $n>1$, for every two-sided $\mathrm{o}$-ideal a, there exists a member $L$ of $\mathfrak{L}(\mathfrak{D})$ such that $N(L)=\mathfrak{a}$. 
Proof. Take an arbitrary maximal lattice $M$ in $V$ with the order $\mathfrak{b}$. There exist only a finite number of $\mathfrak{p}$ such that $N\left(M_{\mathfrak{p}}\right) \neq \mathfrak{a}_{\mathfrak{p}}$. For each one of such $\mathfrak{p}$, take a maximal lattice $L^{\mathfrak{p}}$ in $V_{\mathfrak{p}}$ with the order $\mathfrak{o}_{\mathfrak{p}}$, such that $N\left(L^{\mathfrak{p}}\right)=\mathfrak{a}_{\mathfrak{p}}$. This is possible by Propositions 2.6 and 3.5. Put $L^{\mathfrak{p}}=M_{\mathfrak{p}}$ for every $\mathfrak{p}$ such that $N\left(M_{\mathfrak{p}}\right)=\mathfrak{a}_{\mathfrak{p}}$. Then, by Lemma 1 , there exists a $\mathfrak{g}$-lattice $L$ in $V$ such that $L_{\mathfrak{p}}=L^{\mathfrak{p}}$ for any $\mathfrak{p}$. It is clear that $L$ is a member of $\mathfrak{L}(\mathfrak{p})$ and $N(L)=\mathfrak{a}$.

Proposition 4.6. Let $\mathrm{D}$ be a maximal order in $A$. If $n=1, \mathfrak{L}(\mathrm{D})$ consists of only one genus, $\mathfrak{L}(\mathfrak{D})$ itself. If $n>1$, there are exactly $2^{s}$ genera in $\mathfrak{L}(\mathfrak{D})$, where $s$ is the number of prime ideals ramified in $A / F$.

Proof. The $\mathfrak{Q}_{i}$ being as in $\S 4.1$, we have $N(L)=\mathfrak{Q}_{1}^{e_{1}} \ldots \mathfrak{Q}_{s}^{e_{s}} \cdot \mathfrak{a}$ for every $L \in \mathfrak{R}(\mathfrak{D})$, where $e_{i}=0$ or 1 , and $\mathfrak{a}$ is a $\mathfrak{g}$-ideal. By Proposition 2.11 and Proposition 3.7, the genus of $L$ is determined only by $\left\{e_{1}, \cdots, e_{s}\right\}$. This together with Proposition 4.5 proves our proposition.

We denote, for any set of integers $\left\{e_{1}, \cdots, e_{s}\right\}$ such that $e_{i}=0$ or 1 , by $\mathcal{L}\left(\mathfrak{0} ;\left\{e_{i}\right\}\right)$ the genus of $L$ such that $N(L)=\prod_{i=1}^{s} \mathfrak{\Omega}_{i}^{e_{i}} \cdot \mathfrak{a}$ with an ideal $\mathfrak{a}$ of $F$. We call especially $\mathfrak{L}(\mathfrak{D} ;\{0, \cdots, 0\})$ the principal genus with the order $\mathfrak{D}$ and denote it by $R_{0}(0)$.

Fix a member $L$ of $\mathfrak{L}(\mathfrak{D})$ and define $\mathfrak{H}_{L}$ as in $\S 4.3$. For every element $\xi$ $=\left(\xi_{p}, \xi_{k}\right)$ of the adele-group $\&$, put $L \xi=\bigcap_{p}\left(L_{p} \xi_{p} \cap V\right)$. By Lemma 1.1, $L \xi$ is a g-lattice in $V$; and $(L \xi)_{\mathfrak{p}}=L_{p} \xi_{\mathrm{p}}$. By Propositions 2.2, 2.3, 2.4, we see that $L \xi$ is a member of $\mathcal{L}(\mathrm{D})$. Further, by our definition, $L \xi$ belongs to the same genus as $L$. Conversely, if $M$ is a maximal lattice belonging to the same genus as $L$, we can find an element $\xi$ of $\$$ such that $L \xi=M$. If $\xi \in G$, the notation $L \xi$ is just the same as the transform of $L$ by $\xi$; so there is no fear of confusion. We have $L \xi=L \eta$ if and only if $\mathfrak{H}_{L} \xi=\mathfrak{U}_{L} \eta$. Therefore, the mapping $\xi \rightarrow L \xi$ gives a one-to-one mapping of $\mathfrak{H}_{L} \backslash \mathfrak{S}$ onto the genus of $L$. Moreover, we note that this gives a one-to-one correspondence between $\mathfrak{H}_{L} \backslash \mathbb{S} / G$ and the classes in the genus. By the fact remarked at the end of $\S 4.3$, this implies that each genus consists of a finite number of classes. Further, by Proposition 2.5, we observe that the number of classes in $\mathfrak{R}\left(\mathfrak{D} ;\left\{e_{i}\right\}\right)$ depends only on $\left\{e_{i}\right\}$ and is independent of the choice of $\mathfrak{D}$.

4.5. An existence theorem in the case $\boldsymbol{n}=\mathbf{2}$. Let $A$ be a quaternion algebra over $F$. We denote by $\mathfrak{q}_{1}, \cdots, \mathfrak{q}_{s}$ all the prime ideals of $F$ which are ramified in $A / F$, and by $\mathfrak{p}_{\infty 1}, \cdots, \mathfrak{p}_{\infty u}$ all the infinite prime spots of $F$ which are ramified in $A$. We put

$$
\mathfrak{D}=\prod_{h=1}^{s} \mathfrak{q}_{h}, \quad \mathfrak{u}=\prod_{\kappa=1}^{u} \mathfrak{p}_{\infty_{\kappa}} .
$$

Let $V$ be an $A$-space of dimension $n$ and $f$ a non-degenerate $Q$-hermitian form on $V$. Fix a maximal order $\mathfrak{D}$ in $A$; and for each $\mathfrak{q}_{h}$, let $\mathfrak{Q}_{h}$ be the prime 
$\mathfrak{D}$-ideal such that $\mathfrak{Q}_{h}^{2}=\mathfrak{q}_{h}$. We put $\mathfrak{g}_{\mathfrak{p}} \mathfrak{p}=\mathfrak{p}$ for every prime ideal $\mathfrak{p}$ of $F$, and $\mathfrak{D}_{\mathfrak{q}_{h}} \mathfrak{Q}_{h}=\mathfrak{Q}_{h}$ for every $h$, when there is no fear of confusion.

LEMMA 4.7. Let $\mathfrak{p}$ be a prime ideal of $F$ which is unramified in $A / F$. Let $\mathfrak{o}_{\mathfrak{p}}$ be a maximal order in $A_{\mathfrak{p}}$. Let a be a regular element of $A_{\mathfrak{p}}$ and $\delta$ be an element of $\mathfrak{g}_{\mathfrak{p}}$. Then there exists an element $d$ of $\mathrm{o}_{\mathfrak{p}} \cap a \mathrm{0}_{\mathrm{p}} a^{-1}$ such that $N(d)=\delta$.

PROOF. We may assume that $A_{\mathfrak{p}}=M_{2}\left(F_{\mathfrak{p}}\right)$ and $\mathfrak{D}_{\mathfrak{p}}=M_{2}\left(\mathfrak{g}_{\mathfrak{p}}\right)$. Then we can find units $\varepsilon, \eta$ of $\mathfrak{o}_{\mathfrak{p}}$ such that $\varepsilon a \eta=\left(\begin{array}{cc}\alpha & 0 \\ 0 & \beta\end{array}\right)$ with $\alpha, \beta \in F_{\mathfrak{p}}$. Put $d=\varepsilon^{-1}\left(\begin{array}{ll}1 & 0 \\ 0 & \delta\end{array}\right) \varepsilon$. Then we have $N(d)=\delta$ and $d \in \mathfrak{p}_{\text {p. }}$. Further we get

$$
a^{-1} d a=a^{-1} \varepsilon^{-1}\left(\begin{array}{ll}
1 & 0 \\
0 & \delta
\end{array}\right) \varepsilon \alpha=\eta\left(\begin{array}{cc}
\alpha & 0 \\
0 & \beta
\end{array}\right)^{-1}\left(\begin{array}{cc}
1 & 0 \\
0 & \delta
\end{array}\right)\left(\begin{array}{cc}
\alpha & 0 \\
0 & \beta
\end{array}\right) \eta^{-1}=\eta\left(\begin{array}{cc}
1 & 0 \\
0 & \delta
\end{array}\right) \eta^{-1} \in \mathfrak{b}_{\mathfrak{p}},
$$

so that $d \in a \mathrm{p}_{\mathrm{p}} a^{-1}$, which completes the proof.

Proposition 4.8. Suppose that $A$ is indefinite and $n=2$. Let $L$ be a $\mathfrak{g}$-lattice in $V$ written in the form $L=\mathfrak{o} x+\mathfrak{c}^{-1} y$, where $\mathrm{c}$ is an integral right $\mathrm{D}$-ideal, $f(x, x)$ $=1, f(x, y)=0, f(y, y)=\gamma, N(\mathfrak{c})=r \mathfrak{g}$ with an element $r$ of g. Suppose that $r$ is prime to $\mathrm{d}$. Let $\mathfrak{p}_{1}, \cdots, \mathfrak{p}_{r}$ be distinct prime ideals which are prime to $\mathfrak{b}$, and let $\alpha$ be a non-zero element of $\mathrm{g}$ such that $\alpha \equiv 1 \bmod \mathfrak{p}_{\alpha_{n}}$ whenever $\gamma \equiv 1 \bmod \mathfrak{p}_{\infty_{n}}$ for $1 \leqq \kappa \leqq u$. Put $\mathfrak{g}_{\mathfrak{p}_{i}} \alpha=\mathfrak{p}_{i}^{\mu_{i}}$ for $1 \leqq i \leqq r$ and $\mathfrak{g}_{\mathfrak{q}_{h}} \alpha=\mathfrak{q}_{h}^{\lambda_{h}}$ for $1 \leqq h \leqq s$. Let $\xi_{i}, \eta_{i}$, for $1 \leqq i \leqq r$, and $\nu_{h}$, for $1 \leqq h \leqq s$, be integers such that

$$
0 \leqq \xi_{i} \leqq \eta_{i} \leqq \mu_{i}-\eta_{i} \leqq \mu_{i}-\xi_{i} \leqq \mu_{i}, \quad 0 \leqq \nu_{h} \leqq \lambda_{h} .
$$

Then there exists an element $\sigma$ of $G(V, f)$ such that $L \sigma \subset L, N(\sigma)=\alpha$,

$$
\begin{array}{ll}
\left\{L_{\mathfrak{p}_{i}}: L_{\mathfrak{p}_{i}} \sigma\right\}=\left\{\mathfrak{p}_{i}^{\hat{\xi}_{i}}, \mathfrak{p}_{i}^{\eta_{i}}, \mathfrak{p}_{i}^{\mu_{i}-\xi_{i}}, \mathfrak{p}_{i}^{\mu_{i}-\eta_{i}}\right\} & \text { for } 1 \leqq i \leqq r, \\
\left\{L_{\mathfrak{q}_{h}}: L_{\mathfrak{q}_{h}} \sigma\right\}=\left\{\mathfrak{Q}_{h}^{\nu_{h}}, \mathfrak{Q}_{h}^{2 \lambda_{h}-\nu_{h}}\right\} & \text { for } 1 \leqq h \leqq s .
\end{array}
$$

Proof. For simplicity, we denote the indices $\mathfrak{p}_{i}$ and $\mathfrak{q}_{h}$ respectively by $i$ and $h$; for example, $L_{i}$ means $L_{p_{i}}$ and $g_{h}$ means $g_{q_{h}}$. By Proposition 2.6, $L$ is maximal and $N(L)=\mathfrak{b}$. Now, for each $\mathfrak{p}_{i}$, we identify $\mathfrak{p}_{i}$ with $M_{2}\left(\mathfrak{g}_{i}\right)$, and fix an element $\pi_{i}$ of $\mathfrak{g}$ such that $\mathfrak{p}_{i}=\mathfrak{g}_{i} \pi_{i}$. Put $\mathfrak{g}_{i} \gamma=\mathfrak{p}_{i}^{e_{i}}$. Without any loss of generality, we may assume that $\mathfrak{c}_{i}^{-1}$ is written in the form $\mathfrak{c}_{i}^{-1}=\left(\begin{array}{ll}\mathfrak{p}_{i}^{-c_{i}} & \mathfrak{p}_{i}^{-d_{i}} \\ \mathfrak{p}_{i}^{-c_{i}} & \mathfrak{p}_{i}^{-d_{i}}\end{array}\right)$, where $c_{i}$ and $d_{i}$ are integers such that $c_{i} \geqq d_{i} \geqq 0$ and $c_{i}+d_{i}=e_{i}$. Consider the idealclass modulo $\mathfrak{u}$ containing the inverse of the ideal

$$
\prod_{i=1}^{r} \mathfrak{p}_{i}^{2} \mu_{i}+2+c_{i}-d_{i}+\eta_{i}-\xi_{i} \prod_{h=1}^{s} \mathfrak{q}_{h}^{\nu_{h}}
$$

We can find an integral ideal $\mathfrak{a}$ in that class which is prime to $\gamma \alpha \prod_{i=1}^{r} \mathfrak{p}_{i} \cdot \delta$. We get then

$$
\mathfrak{a} \cdot \prod_{i=1}^{r} \mathfrak{p}_{i}^{2 \mu_{i}+2+c_{i}-d_{i}+\eta_{i}-\xi_{i}} \prod_{h=1}^{s} \mathfrak{q}_{h}^{\nu}=(\beta), \quad \beta \equiv 1 \bmod \mathfrak{u}
$$

for an element $\beta$ of $\mathrm{g}$. By Lemma 4.3, there exists an element $a_{1}$ of $\mathfrak{D}$ such that $N\left(a_{1}\right)=\beta$ and 


$$
a_{1} \equiv\left(\begin{array}{cc}
\pi_{i}^{\mu_{i}+1} & 0 \\
0 & \beta \pi_{i}^{-\mu_{i-1}}
\end{array}\right) \quad \bmod \beta \mathfrak{p}_{i}^{\mu_{i}+e_{i}+1} \mathfrak{o}_{i} \quad(1 \leqq i \leqq r) .
$$

Let $\varepsilon$ be a unit of $F$ such that $\alpha\left(1-\varepsilon^{2}\right) \equiv 1 \bmod u$. Such an $\varepsilon$ really exists, because $\mathfrak{u}$ is not the product of all the infinite prime spots of $F$. Then, by our assumption on $\alpha$, we have

$$
\alpha-\varepsilon^{2 m} N\left(a_{1}\right) \gamma \equiv 1 \quad \bmod \mathfrak{}
$$

for a suitably large integer $m$. Fix such an $m$. By Lemma 4.3, there exists an element $b_{1}$ of $\mathfrak{p}$ such that $N\left(b_{1}\right)=\alpha-\varepsilon^{2 m} N\left(a_{1}\right) \gamma$. By our choice of $\beta$, we observe that $\mathfrak{g}_{i} N\left(b_{1}\right)=\mathfrak{p}_{i}^{\mu_{i}}$ for $1 \leqq i \leqq r$. Put $a=\varepsilon^{m} a_{1}$. For every prime ideal $\mathfrak{r}$ of $F$, let $\bar{D}_{\mathfrak{x}}$ denote the right order of $\left(\mathfrak{c}^{-1} a^{-1}\right)_{\mathfrak{r}}$. Let $\{\mathfrak{r}\}$ be the set of prime ideals $\mathfrak{r}$ such that $\left(\mathfrak{r}, \mathfrak{b} \cdot \prod_{i=1}^{r} \mathfrak{p}_{i}\right)=1, \overline{\mathfrak{D}}_{\mathfrak{r}} \neq \mathfrak{D}_{\mathfrak{r}}$. Obviously $\{\mathfrak{r}\}$ is a finite set. For each $\mathfrak{r}$, take an element $b_{\mathfrak{r}}$ of $\overline{\mathfrak{D}}_{\mathfrak{r}} \cap \mathfrak{D}_{\mathfrak{r}}$ such that $N\left(b_{\mathfrak{r}}\right)=N\left(b_{1}\right)$. This is possible by virtue of Lemma 4.7. Now by Lemma 4.3, we can find an element $b$ of $\mathfrak{D}$ such that $N(b)=N\left(b_{1}\right), b \equiv b_{\mathfrak{x}} \bmod \left(\overline{\mathfrak{D}}_{\mathfrak{x}} \cap \mathfrak{D}_{\mathfrak{r}}\right)$ for $\mathfrak{r} \in\{\mathfrak{r}\}$, and

$$
b \equiv\left(\begin{array}{cc}
0 & \pi_{i}^{\xi_{i}} \\
-N\left(b_{1}\right) \pi_{i}^{-\xi_{i}} & 0
\end{array}\right) \quad \bmod \beta \mathfrak{p}_{i}^{\mu_{i}+e_{i}+1_{\mathfrak{o}_{i}}} \quad(1 \leqq i \leqq r) .
$$

Then we have $N(b)+\gamma N(a)=\alpha$, and by (11) and (12),

$$
a^{-1} b a=a_{1}^{-1} b a_{1}=\beta^{-1} a_{1}^{\prime} b a_{1} \equiv\left(\begin{array}{cc}
0 & \beta \pi_{i}^{\xi_{i}-2 \mu_{i}-2} \\
-N(b) \beta^{-1} \pi_{i}^{-\xi} \xi_{i+2} \mu_{i+2} & 0
\end{array}\right) \bmod \mathfrak{p}_{i}^{\mu_{i}+e_{i}+\mathfrak{v}_{i}},
$$

so that

$$
a^{-1} b^{\prime} a \equiv\left(\begin{array}{cc}
0 & \theta \pi_{i}^{\eta_{i}+c_{i}-d_{i}} \\
\psi \pi_{i}^{\mu_{i}-\eta_{i-c_{i}+d_{i}}} & 0
\end{array}\right) \bmod \mathfrak{p}_{i}^{\mu_{i}+e_{i}+1_{\mathfrak{b}_{i}}}
$$

with units $\theta$ and $\psi$ of $\mathrm{g}_{i}$. It follows that

$$
\mathfrak{c}_{i}^{-1}\left(a^{-1} b^{\prime} a\right)=\left(\begin{array}{ll}
\mathfrak{p}_{i}^{\mu_{i}-\eta_{i}-c_{i}} & \mathfrak{p}_{i}^{\eta_{i}-d_{i}} \\
\mathfrak{p}_{i}^{\mu_{i}-\eta_{i}-c_{i}} & \mathfrak{p}_{i}^{\eta_{i}-d_{i}}
\end{array}\right) \text {. }
$$

Hence we have $c_{i}^{-1}\left(a^{-1} b^{\prime} a\right) \subset c_{i}^{-1}$. By our choice of $b_{\mathfrak{x}}$, we have $b^{\prime} \in \overline{\mathfrak{D}}_{\mathfrak{x}}$ for every $\mathfrak{r}$ such that $\left(\mathfrak{r}, \mathfrak{b} \cdot \prod_{i=1}^{r} \mathfrak{p}_{i}\right)=1$, and hence $\mathfrak{c}_{\mathfrak{r}}^{-1}\left(a^{-1} b^{\prime} a\right) \subset \mathfrak{c}_{\mathfrak{r}}^{-1}$ for any such $\mathfrak{r}$. Further it is obvious that $\mathfrak{c}_{h}^{-1}\left(a^{-1} b^{\prime} a\right) \subset c_{h}^{-1}$. Therefore, we have

$$
c^{-1}\left(a^{-1} b^{\prime} a\right) \subset c^{-1} \text {. }
$$

As $a \in \mathfrak{D} \subset \mathfrak{c}^{-1}$ and $N(\mathfrak{c})=r g$, we have

Moreover, by [11) we have

$$
\mathfrak{c}^{-1} \gamma a^{\prime} \subset \mathfrak{D} \text {. }
$$

Now define an element $\sigma$ of $E(V, A)$ by

$$
x \sigma=b x+a y, \quad y \sigma=-\gamma a^{\prime} x+a^{-1} b^{\prime} a y .
$$

By the relation $N(b)+\gamma N(a)=\alpha$, we can easily verify that $\sigma \in G(V, f)$ and 
$N(\sigma)=\alpha$. Further by (15), $\left(15^{\prime}\right)$, we have $L \sigma \subset L . \quad$ By Proposition 2.7, we have $\mathfrak{p}_{i}^{\mu_{i}} L_{i}=\alpha L_{i} \subset L_{i} \sigma$, so that

$$
\mathfrak{p}_{i}^{\mu}{ }^{\mu+1} L_{i} \subset \mathfrak{p}_{i} L_{i} \sigma \text {. }
$$

Put $M_{i}=\mathfrak{p}_{i} b x+c_{i}^{-1} a^{-1} b^{\prime} a y$. Then we have, by (11), (16), (17), (18) and by Lemma $1.2, M_{i}=L_{i} \sigma$, so that $L_{i} / L_{i} \sigma=L_{i} / M_{i} \cong \mathfrak{p}_{i} / \mathfrak{o}_{i} b+\mathfrak{c}_{i}^{-1} / \mathfrak{c}_{i}^{-1} a^{-1} b^{\prime} a$ (as $\mathfrak{o}_{i}$-modules). By (12) and (14), $L_{i} / L_{i} \sigma$ has the desired elementary divisors. Let us now consider $\mathfrak{q}_{h}$ for $1 \leqq h \leqq s$. As $N(\sigma)=\alpha$ and $\mathfrak{b}_{h} \alpha=\mathfrak{Q}_{h}^{2 \lambda_{h}}$, we have, by Proposition 2.7, $L_{h} \sigma \supset \mathfrak{Q}_{h}^{2 \lambda_{h}} L_{h}$. As $N\left(a_{1}\right)=\beta$ and $\mathfrak{g}_{h} \beta=q_{h}^{\nu} h$, we have $\mathfrak{p}_{h} a=\mathfrak{Q}_{h}^{\nu} h$. If $\lambda_{h}=0$, we have $L_{h} \sigma=L_{h}$. Suppose that $\lambda_{h}>0$. If $\nu_{h}=\lambda_{h}$, we have $\mathfrak{b}_{h} a=\mathfrak{Q}_{h} \lambda_{h}$ and hence $N(b)=\alpha-\gamma N(a) \in \mathfrak{Q}_{h}^{2 \lambda_{h}}$. It follows that $a, b,-\gamma a^{\prime}, a^{-1} b^{\prime} a$ are contained in $\mathfrak{Q}_{h}^{\lambda_{h}}$. Hence we have $x \sigma, y \sigma \in \mathfrak{Q}_{h}^{h} L_{h}$, so that $L_{h} \sigma \subset \mathfrak{Q}_{h}^{\lambda_{h}} L_{h}$. As $L_{h} \sigma$ is maximal and $N\left(L_{h} \sigma\right)=\mathfrak{Q}_{h}^{\lambda_{h}}=N\left(\mathfrak{Q}_{h}^{\lambda_{h}} L_{h}\right)$, we must have $L_{h} \sigma=\mathfrak{Q}_{h}^{\lambda_{h}} L_{h}$. Then $L_{h} / L_{h} \sigma \cong \mathfrak{D}_{h} / \mathfrak{Q}_{h}^{\lambda_{h}}$ $+\mathfrak{D}_{h} / \mathfrak{Q}_{h}^{\lambda_{h}}$. It remains to consider the case $\lambda_{h}>\nu_{h} \geqq 0$. As $N(b)+\gamma N(\alpha)=\alpha, \mathfrak{b}_{h} a$ $=\mathfrak{Q}_{h}^{\nu}, \mathfrak{D}_{h} \alpha=\mathfrak{Q}_{h}^{2 \lambda_{h}}$, we must have $\mathfrak{D}_{h} b=\mathfrak{Q}_{h}^{\nu_{h}}$. It follows that $\dot{a}^{\prime} b^{-1}$ is a unit of $\mathfrak{b}_{h}$. We note that

$$
\begin{aligned}
& y \sigma+\gamma\left(a^{\prime} b^{-1}\right) x \sigma=\left(a^{-1} b^{\prime} a+\gamma a^{\prime} b^{-1} a\right) y=a^{-1} b^{-1}\left(b b^{\prime}+r b a a^{\prime} b^{-1}\right) a y \\
= & a^{-1} b^{-1}(N(b)+\gamma N(a)) a y=\alpha\left(a^{-1} b^{-1} a\right) y .
\end{aligned}
$$

Therefore we have $L_{h} \sigma=\mathfrak{o}_{h} x \sigma+\mathfrak{o}_{h} y \sigma=\mathfrak{o}_{h} x \sigma+\mathfrak{o}_{h} \alpha\left(a^{-1} b^{-1} a\right) y$. On the other hand, as $b^{-1} a$ is a unit of $\mathfrak{p}_{h}$ and as $x=b^{-1} x \sigma-b^{-1} a y$, we have $L_{h}=\mathfrak{p}_{h} x+\mathfrak{p}_{h} y=\mathfrak{p}_{h} b^{-1} x \sigma$ $+\mathfrak{o}_{h} y$. Hence $L_{h} / L_{h} \sigma \cong \mathfrak{o}_{h} b^{-1} / \mathfrak{o}_{h}+\mathfrak{p}_{h} / \mathfrak{p}_{h}\left(a^{-1} b^{-1} a\right) \alpha=\mathfrak{p}_{h} / \mathfrak{Q}_{h}^{\nu_{h}}+\mathfrak{o}_{h} / \mathfrak{Q}_{h}^{2 \lambda_{h}-\nu_{h}}$ (as $\mathfrak{o}_{h}^{-}$ modules). This completes our proof.

4.6. Global approximation theorem. As in $\S 4.2$, we define $\nu_{k}(f)$ for $1 \leqq \kappa \leqq u$, and reorder the $\mathfrak{p}_{\times_{\kappa}}$ so that $\nu_{\kappa}(f) \neq n / 2$ for $1 \leqq \kappa \leqq t$ and $\nu_{\kappa}(f)=n / 2$ for $t<\kappa \leqq u$. Put

$$
\mathrm{t}=\mathrm{t}_{f}=\prod_{\kappa=1}^{t} \mathfrak{p}_{\infty \kappa} .
$$

For every $\sigma \in G(V, f)$, we have $N(\sigma) \equiv 1 \bmod$ t. If $n$ is odd, we have $t=u$ and $\mathrm{t}=\mathfrak{u}$.

Now we are ready to state and prove our main theorems.

Theorem 1. Suppose that $A$ is indefinite. Let $L$ be a maximal lattice belonging to the principal genus $\mathfrak{D}_{0}(\mathfrak{D})$. Let $\mathfrak{p}_{1}, \cdots, \mathfrak{p}_{r}$ be prime ideals of $F$; and let $\sigma_{i}$, for each $i$, be an element of $G\left(V_{p_{i}}, f\right)$ such that $L_{p_{i}} \sigma_{i} \subset L_{p_{i}}$. Let $\alpha$ be an element of g. Suppose that

$$
\begin{aligned}
\alpha^{-1} N\left(\sigma_{i}\right) & \equiv 1 \quad \bmod \mathfrak{p}_{i}^{\lambda i}, \quad(1 \leqq i \leqq r), \\
\alpha & \equiv 1 \quad \bmod \mathrm{t},
\end{aligned}
$$

where the $\lambda_{i}$ are positive integers. Then there exists an element $\sigma$ of $G(V, f)$ such that $L \sigma \subset L, N(\sigma)=\alpha, L_{p_{i}}\left(\sigma-\sigma_{i}\right) \subset \mathfrak{p}_{i}^{\lambda i} L_{p_{i}}(1 \leqq i \leqq r)$. 
We prove Theorem 1 in several steps. For simplicity, we denote $L_{\mathfrak{p}_{i}}, \mathfrak{g}_{\mathfrak{p}_{i}}$, etc. by $L_{i}, \mathfrak{g}_{i}$, etc.

ASSERTION 1. If Theorem 1 is proved when $N(L)=\mathfrak{D}$ for every maximal order $\mathfrak{n}$, then it is true for any $L$ belonging to the principal genus.

In fact, if $L$ belongs to $\mathfrak{R}_{0}(\mathfrak{D})$, we can find a left $\mathfrak{D}$-ideal $\mathfrak{x}$ such that $N(L)$ $=N(\mathfrak{x}) \mathfrak{0}$. Then by Proposition 2.5, $\mathfrak{x}^{-1} L$ is a maximal lattice, and $N\left(\mathfrak{x}^{-1} L\right)=\mathfrak{b}_{1}$, where $\mathfrak{b}_{1}$ is the right order of $\mathfrak{x}$. We see easily that if Theorem 1 is true for $\mathfrak{x}^{-1} L$, then it is true for $L$.

AsSERTION 2. If Theorem 1 is true for $L$, then, for every $\tau \in G(V, f)$, Theorem 1 is true for $L \tau$.

This is clear.

ASSERTION 3. If Theorem 1 is proved for a certain $L^{0} \in \mathbb{R}_{0}(\mathrm{D})$ such that $N\left(L^{0}\right)=\mathfrak{p}$, then for any $L \in \mathfrak{R}_{0}(\mathfrak{n})$ such that $N(L)=\mathfrak{b}$, we have $L=L^{0} \tau$ for an element $\tau \in G^{0}(V, f)$.

Let $\beta$ be a non-zero element of $g$ such that $\beta L \subset L^{0}$. Take an integral $\mathfrak{g}$-ideal $\mathfrak{a}$ such that $\beta L \supset \mathfrak{a} L^{0}$. Let $\mathfrak{p}_{1}, \cdots, \mathfrak{p}_{r}$ be the prime factors of $\mathfrak{a}$. By Proposition 2.11 and by Proposition 3.7, there exists, for each $\mathfrak{p}_{i}$, an element $\tau_{i}$ of $G^{0}\left(V_{i}, f\right)$ such that $L_{i}^{0} \tau_{i}=L_{i}$. Put $\sigma_{i}=\beta \tau_{i}$. Then we have $N\left(\sigma_{i}\right)=\beta^{2}$. Applying Theorem 1 to $L^{0}$ and these $\sigma_{i}$, we get an element $\sigma$ of $G(V, f)$ such that $L^{0} \sigma \subset L^{0}, N(\sigma)=\beta^{2}, L_{i}^{0}\left(\sigma-\sigma_{i}\right) \subset \mathfrak{p}_{i} \mathfrak{a} L_{i}^{0}$ for $1 \leqq i \leqq \gamma$. As we have $\mathfrak{p}_{i} \mathfrak{a} L_{i}^{0} \subset \mathfrak{p}_{i} \beta L_{i}$ $=\mathfrak{p}_{i} L_{i}^{0} \sigma_{i}$, we see, from Lemma 1.2, $L_{i}^{0} \sigma=L_{i}^{0} \sigma_{i}=\beta L_{i}$. If $\mathfrak{p}$ is a prime ideal which does not divide $\mathfrak{a}$, we have $L_{p}^{0}=\beta L_{p}$, since $L^{0} \supset \beta L \supset \mathfrak{a} L^{0}$. It follows that $N\left(L_{\mathfrak{p}}^{0}\right)=\beta^{2} N\left(L_{p}\right)$, and hence $\beta$ is a $\mathfrak{p}$-unit. As $L^{0} \sigma \subset L^{0}$ and $N(\sigma)=\beta^{2}$, we have $L_{p}^{0} \sigma=L_{p}^{0}=\beta L_{p}$. Therefore, we have $L_{p}^{0} \sigma=\beta L_{\mathfrak{p}}$ for every prime ideal $\mathfrak{p}$ of $F$, so that $L^{0} \sigma=\beta L$. Putting $\tau=\beta^{-1} \sigma$, we get $L^{0} \tau=L$.

ASSERTION 4. In order to prove Theorem 1, we may exchange the $Q$ hermitian form $f$ for $\theta f$ for any non-zero element $\theta$ of $F$.

In fact, put $g(x, y)=\theta f(x, y)$ for $(x, y) \in V \times V$. Then $g$ is a non-degenerate $Q$-hermitian form. We see easily $G(V, f)=G(V, g), G^{0}(V, f)=G^{0}(V, g)$, and, for every $\sigma \in G(V, f), N(\sigma)$ is common for $f$ and $g$. Further, for every $g$-lattice $L$ in $V$, we have $N_{g}(L)=\theta N_{f}(L)$. When $L$ is normal, $L$ is maximal with respect to $f$ if and only if $L$ is maximal with respect to $g$. The genera and the classes of normal maximal lattices do not change by exchanging $f$ for $g$. Finally we note that $t_{f}=t_{g}$. Therefore we get Assertion 4 .

Now we proceed by induction on $n$. If $n=1$, Theorem 1 is just a restatement of Lemma 4.3. Assume that $n>1$ and Theorem 1 is true for $\operatorname{dim}_{A} V<n$.

ASSERTION 5. If $N(L)=\mathfrak{p}$ and $L$ contains an element $x$ such that $f(x, x)=1$, then Theorem 1 is true for this $L$ and for $\alpha=1$.

As $N\left(\sigma_{i}\right) \equiv 1 \bmod p_{i}^{\lambda i}$ and $N(L)=\mathfrak{p}$, we have $f\left(u \sigma_{i}, v \sigma_{i}\right) \equiv f(u, v) \bmod \mathfrak{p}_{i}^{\lambda i_{i}}$ for 
$u, v \in L . \quad$ By Proposition 3.13, there exists an element $\tau_{i}$ of $G\left(V_{i}, f\right)$ such that $N\left(\tau_{i}\right)=1, L_{i} \tau_{i}=L_{i}, L_{i}\left(\sigma_{i}-\tau_{i}\right) \subset p_{i}^{\lambda i} L_{i}$. Put

$$
W=\{v \in V \mid f(x, v)=0\}, \quad M=W \cap L .
$$

Then we have $V=A x+W, L=\mathfrak{p} x+M$; and $M$ is a maximal lattice in $W$ such that $N(M)=$ o. Put $x \tau_{i}=a_{i} x+y_{i}$ with $a_{i} \in \mathrm{p}_{i}$ and $y_{i} \in M_{i} . \quad$ As $f\left(x \tau_{i}, x \tau_{i}\right)=f(x, x)$ $=1$, we have $N\left(a_{i}\right)+f\left(y_{i}, y_{i}\right)=1$. Now reorder the $\mathfrak{p}_{i}$ so that $N\left(a_{i}\right) \neq 0$ for $1 \leqq i \leqq h, N\left(a_{i}\right)=0$ for $h<i \leqq r$, where $h$ is an integer such that $0 \leqq h \leqq r$. Put $\mathrm{g}_{i} N\left(a_{i}\right)=\mathfrak{p}_{i}^{\mu_{i}}$ for $1 \leqq i \leqq h$. We can find a regular element $a$ of $A$ such that. $a \in \mathfrak{D}$, and

We have then

$$
\begin{array}{lll}
a \equiv a_{i} \bmod \mathfrak{p}_{i} \uparrow_{i}^{\lambda_{i}+\mu_{i}} & \text { for } 1 \leqq i \leqq h \\
a \equiv a_{i} \bmod \mathfrak{p}_{i} \uparrow_{i}^{\lambda_{i}} & \text { for } h<i \leqq r .
\end{array}
$$

$$
\begin{aligned}
& N(a) \equiv N\left(a_{i}\right) \quad \bmod \mathfrak{p}_{i}^{\lambda_{i}+\mu_{i}} \quad \text { for } \quad 1 \leqq i \leqq h, \\
& N(a) \equiv 0 \quad \bmod \mathfrak{p}_{i}^{\lambda i} \quad \text { for } h<i \leqq r \text {, }
\end{aligned}
$$

and hence $\mathfrak{g}_{i} N(a)=\mathfrak{p}_{i}^{\mu_{i}}$ for $1 \leqq i \leqq h$. Put $\mathfrak{g}_{i} N(a)=\mathfrak{p}_{i}^{\mu_{i}}$ for $i>h$. We have then $\mu_{i} \geqq \lambda_{i} \geqq 1$ for $i>h$. Now, as $1-N(\alpha) \equiv 1 \bmod \mathfrak{p}_{i}^{\mu_{i}}$, we can find, by Proposition 3.2 , for each $i>h$, an element $\varepsilon_{i}$ of $\mathfrak{p}_{i}$ such that $\varepsilon_{i} \equiv 1 \bmod \mathfrak{p}_{i} \mathfrak{p}_{i}^{\mu_{i}}, N\left(\varepsilon_{i}\right)=1-N(a)$. Take an element $y$ of $M$ so that

$$
\begin{aligned}
& y \equiv y_{i} \quad \bmod \mathfrak{p}_{i}^{\lambda i+\mu_{i}} M_{i} \quad \text { for } \quad 1 \leqq i \leqq h, \\
& y \equiv \varepsilon_{i} y_{i} \quad \bmod \mathfrak{p}_{i}^{\lambda i+\mu_{i}} M_{i} \quad \text { for } \quad h<i \leqq r .
\end{aligned}
$$

Then we can easily verify that $f(y, y) \equiv 1-N(a) \bmod \mathfrak{p}_{i}^{\lambda_{i}+\mu_{i}}$ for every $i$. Since $\mathfrak{u}$ is not the product of all infinite prime spots of $F$, the projection of the set $\left\{\beta \mid \beta=1+\xi, \xi \in \prod_{i=1}^{r} \mathfrak{p}_{i}^{\mu_{i}+\lambda_{i}}\right\}$ on $F_{1} \times \cdots \times F_{u}$ is dense. Hence there exists an element $\beta$ of $\mathfrak{g}$ such that $1-\beta^{2} \equiv 1 \bmod \mathfrak{t t}, \beta \equiv 1 \bmod \prod_{i=1}^{r} \mathfrak{p}_{i}^{\mu_{i}+\lambda_{i}}$. For a suitably large integer $k, 1-\beta^{2 k} f(y, y) \equiv 1 \bmod \mathfrak{u}$. Put $w=\beta^{k} y$ for such an integer $k$. Then we have

$$
\begin{gathered}
w \equiv y \quad \bmod \prod_{i=1}^{r} \mathfrak{p}_{i}^{\lambda_{i}+\mu_{i}} M, \\
1-f(w, w) \equiv 1-f(y, y) \equiv N(a) \quad \bmod \prod_{i=1}^{r} \mathfrak{p}_{i}^{\lambda_{i}+\mu_{i}}, \\
1-f(w, w) \equiv 1 \quad \bmod \mathfrak{u} .
\end{gathered}
$$

As $N(a) \mathfrak{g}_{i}=\mathfrak{p}_{i}^{\mu_{i}}$, we have $N(a)^{-1}(1-f(w, w)) \equiv 1 \bmod \prod_{i=1}^{r} \mathfrak{p}_{i}^{\lambda_{i}}$. Therefore, by Lemma. 4.3 , there exists an element $b$ of $\mathfrak{D}$ such that

$$
N(b)=1-f(w, w), \quad b \equiv \alpha \bmod \prod_{i=1}^{r} p_{i}^{\lambda} i_{\mathfrak{D}} .
$$

Put $u=b x+w$. Then $f(u, u)=N(b)+f(w, w)=1$, and 


$$
u \equiv a x+y \equiv a_{i} x+y_{i}=x \tau_{i} \quad \bmod \mathfrak{p}_{i}^{\lambda i} L_{i} \quad(1 \leqq i \leqq r)
$$

Hence, putting

$$
U=\{z \in V \mid f(u, z)=0\}, \quad K=U \cap L,
$$

we have $V=A u+U, L=\mathfrak{v} u+K$; and $K$ is a maximal lattice in $U$ such that $N(K)=$ p. By Lemma 4.4, $(U, f)$ and $(W, f)$ are isomorphic. Therefore we can find an element $\rho$ of $G^{0}(V, f)$ such that $x \rho=u, W \rho=U$. We see easily that $M \rho$ is a maximal lattice in $U$ and $N(M \rho)=\mathfrak{p}$. By our induction assumption and Assertion 3, there exists an element $\varphi$ of $G^{0}(U, f)$ such that $M \rho \varphi=K$. Exchanging $\rho$ for $\rho \varphi$ on $W$, we may assume that $M \rho=K$ for $\rho$ itself. Then we have $L \rho=L$, and $x \tau_{i} \rho^{-1} \equiv x \bmod \mathfrak{p}_{i}^{\lambda_{i}} L_{i}$. For every $z \in W_{i}$, denote by $z \psi_{i}$ the projection of $z \tau_{i} \rho^{-1}$ onto $W_{i}$ defined by the decomposition $V_{i}=A_{i} x+W_{i}$. Then $\psi_{i}$ can be considered as an element of $E\left(W_{i}, A_{i}\right)$. If $z \in M_{i}$, we have $f\left(z \tau_{i} \rho^{-1}, x\right) \equiv f\left(z \tau_{i} \rho^{-1}, x \tau_{i} \rho^{-1}\right)=f(z, x)=0 \bmod p_{i}^{\lambda} i_{i} . \quad$ It follows that $M_{i}\left(\tau_{i} \rho^{-1}-\psi_{i}\right)$ $\subset \mathfrak{p}_{i}^{\lambda i} \mathfrak{D}_{i} x$, and hence $f\left(z_{1} \psi_{i}, z_{2} \psi_{i}\right) \equiv f\left(z_{1}, z_{2}\right) \bmod \mathfrak{p}_{i}^{\lambda} \mathfrak{D}_{i}$ for $z_{1} \in M_{i}, z_{2} \in M_{i}$. By Proposition 3.13, there exists an element $\theta_{i}$ of $G^{0}\left(W_{i}, f\right)$ such that $M_{i} \theta_{i} \subset M_{i}$ and $M_{i}\left(\psi_{i}-\theta_{i}\right) \subset p_{i}^{\lambda i} M_{i}$. Applying our induction assumption to $M$ and the $\theta_{i}$, we find an element $\theta$ of $G^{0}(W, f)$ such that $M \theta \subset M, M_{i}\left(\theta-\theta_{i}\right) \subset \mathfrak{p}_{i}^{i} M_{i}$ for $1 \leqq i \leqq r$. Define an element $\sigma$ of $E(V, A)$ by $x \sigma=u, z \sigma=z \theta \rho$ for $z \in W$. Then we have $\sigma \in G(V, f)$ and $N(\sigma)=1, L \sigma \subset L$. Further, we have

$$
x \sigma=u \equiv x \tau_{i} \equiv x \sigma_{i} \quad \bmod \mathfrak{p}_{i}^{\lambda i} L_{i} \quad(1 \leqq i \leqq r),
$$

and if $z \in M_{i}$,

$$
z \sigma=z \theta \rho \equiv z \theta_{i} \rho \equiv z \psi_{i} \rho \equiv z \tau_{i} \equiv z \sigma_{i} \quad \bmod \mathfrak{p}_{i}^{\lambda} L_{i} \quad(1 \leqq i \leqq \gamma) .
$$

Therefore $L_{i}\left(\sigma-\sigma_{i}\right) \subset \mathfrak{p}_{i}^{\lambda} L_{i}$. This completes the proof of Assertion 5 .

Proposition 4.9. Let $L$ be a maximal lattice belonging to $\mathfrak{I}_{0}(\mathfrak{D})$. Let $\alpha$ be an element of $\mathfrak{g}$ such that $\alpha \equiv 1 \bmod \mathrm{t}$, and let $\mathfrak{p}_{1}, \cdots, \mathfrak{p}_{r}$ be prime ideals of $F$. Let $\sigma_{i}$, for $1 \leqq i \leqq r$, be an element of $G\left(V_{p_{i}}, f\right)$ such that $N\left(\sigma_{i}\right) g_{p_{i}}=\alpha \mathfrak{g}_{\mathfrak{p}_{i}}, \quad L_{p_{i}} \sigma_{i}$ $\subset L_{p_{i}}$. Then there exists an element $\sigma$ of $G(V, f)$ such that $N(\sigma)=\alpha, L \sigma \subset L$, and $L_{\mathfrak{p}_{i}} / L_{\mathfrak{p}_{i}} \sigma$ is isomorphic to $L_{\mathfrak{p}_{i}} / L_{\mathfrak{p}_{i}} \sigma_{i}$ as $\mathfrak{p}_{\mathfrak{p}_{i}}$-modules for $1 \leqq i \leqq r$.

ASSERTION 6. If Theorem 1 is true, then Proposition 4.9 is true.

In fact, as $N\left(\sigma_{i}\right)^{-1} \alpha$ is a $\mathfrak{p}_{i}$-unit for each $i$, there exists, by Proposition 3.3 and Proposition 3.12, an element $\tau_{i}$ of $G\left(V_{i}, f\right)$ such that $L_{i} \tau_{i}=L_{i}, N\left(\tau_{i}\right)=N\left(\sigma_{i}\right)^{-1} \alpha$. Then we have $L_{i} \tau_{i} \sigma_{i} \subset L_{i}, N\left(\tau_{i} \sigma_{i}\right)=\alpha$. By Theorem 1, there exists an element $\sigma$ of $G(V, f)$ such that $L \sigma \subset L, N(\sigma)=\alpha, L_{i}\left(\sigma-\tau_{i} \sigma_{i}\right) \subset \mathfrak{p}_{i}^{\lambda i+1} L_{i}=\mathfrak{p}_{i} \alpha L_{i}$ for every $i$. By Proposition 2.7, we have $\alpha L_{i} \subset L_{i} \tau_{i} \sigma_{i}$. Therefore, by Lemma 1.2, we have $L_{i} \sigma=L_{i} \tau_{i} \sigma_{i}=L_{i} \sigma_{i}$. This proves our assertion.

Now exchanging $f$ for $\theta f$ with a suitable $\theta$ of $F$, if necessary, we may assume that $\nu_{\kappa}(f)>n / 2$ for $1 \leqq \kappa \leqq t, \nu_{\kappa}(f)=n / 2$ for $\kappa<t \leqq u$. By Assertion 4 , this does not influence the validity of our proof of Theorem 1 .

Assertion 7. There exists a member $L$ of $\Omega_{0}(\mathfrak{D})$ satisfying the following 
conditions: i) $N(L)=\mathfrak{p}$; ii) $L$ contains an element $x$ such that $f(x, x)=1$; iii) Proposition 4.9 is true for $L$.

To prove this, let $U=A x+A y$ be an $A$-space of dimension 2. We can find an element $\gamma$ of $g$ such that $(\gamma, \mathfrak{b})=1, \gamma \equiv 1 \bmod \mathfrak{p}_{\infty_{\kappa}}$ for $1 \leqq \kappa \leqq t, \gamma \equiv-1 \bmod \mathfrak{p}_{\infty_{\kappa}}$ for $t<\kappa \leqq u$. Define a $Q$-hermitian form $f_{0}$ on $U$ by $f_{0}(x, x)=1, f_{0}(x, y)=0$, $f_{0}(y, y)=r$. Now let $W$ be an $A$-space of dimension $n-2$ and $f_{1}$ be a nondegenerate $Q$-hermitian form on $W$ such that $\nu_{k}\left(f_{1}\right)=\nu_{k}(f)-2$ for $1 \leqq \kappa \leqq t$ and $\nu_{k}\left(f_{1}\right)=\nu_{k}(f)-1$ for $t<\kappa \leqq u$. Then, by Lemma 4.4, $(V, f)$ is isomorphic to the direct sum of $\left(U, f_{0}\right)$ and $\left(W, f_{1}\right)$. Therefore, we may assume that $V=U+W$ $=A x+A y+W, W=\{z \in V \mid f(x, z)=f(y, z)=0\}, f=f_{0}$ on $U \times U$ and $f=f_{1}$ on $W \times W$. Further we see easily that $\mathrm{t}\left(f_{1}\right)$ is a factor of $\mathrm{i}(f)$. Let $\mathrm{c}$ be an integral right $\mathfrak{D}$-ideal such that $N(\mathrm{c})=\mathrm{g} r$, and let $M$ be a maximal lattice in $W$ such that $N(M)=\mathfrak{p}$. Put

$$
K=\mathfrak{o x}+\mathrm{c}^{-1} y, \quad L=K+M .
$$

Then, $K$ is a maximal lattice in $U, L$ is a maximal lattice in $V$; and $N(K)$ $=N(L)=\mathfrak{0}$. Now let the notation be as in Proposition 4.9. The structure of the $\mathfrak{D}_{i}$-module $L_{i} / L_{i} \sigma_{i}$ is determined by Proposition 2.12 and Proposition 3.9. In view of those propositions, we can find an element $\tau_{i}$ of $G\left(U_{i}, f_{0}\right)$ and an element $\rho_{i}$ of $G\left(W_{i}, f_{1}\right)$ such that $N\left(\tau_{i}\right)=\alpha, N\left(\rho_{i}\right)=\alpha$, and

$$
L_{i} / L_{i} \sigma_{i} \cong K_{i} / K_{i} \tau_{i} \oplus M_{i} / M_{i} \rho_{i} \quad(1 \leqq i \leqq r),
$$

where $\cong$ means $\mathfrak{o}_{i}$-isomorphism. As $\alpha \equiv 1 \bmod \mathrm{t}(f)$, we have $\alpha \equiv 1 \bmod \mathrm{t}\left(f_{1}\right)$. By Assertion 6 and by our assumption of induction, there exists an element $\rho$ of $G\left(W, f_{1}\right)$ such that $N(\rho)=\alpha, M \rho \subset M$,

$$
M_{i} / M_{i} \rho \cong M_{i} / M_{i} \rho_{i} \quad(1 \leqq i \leqq r) .
$$

By Proposition 4.8, there exists an element $\tau$ of $G\left(U, f_{0}\right)$ such that $N(\tau)=\alpha$, $K \tau \subset K, K_{i} / K_{i} \tau \cong K_{i} / K_{i} \tau_{i}(1 \leqq i \leqq r)$. Define an element $\sigma$ of $E(V, A)$ by $z \sigma=z \tau$ for $z \in U$ and $w \sigma=w \rho$ for $w \in W$. Then it is clear that this $\sigma$ has the required properties of Proposition 4.9. Our assertion is thereby proved.

ASSERTION 8. For every maximal order $\mathfrak{D}$ in $A$, there exists a member $L$. of $\Omega_{0}(\mathfrak{D})$ for which Theorem 1 is true and $N(L)=\mathfrak{D}$.

We take as $L$ the one which satisfies the conditions $\mathrm{i}$-iii) of Assertion 7. By Assertion 5, Theorem 1 is true for $\alpha=1$, for this $L$. Now let the notation be as in Theorem 1. Then $\mathrm{g}_{i} N\left(\sigma_{i}\right)=\mathrm{g}_{i} \alpha$. By Assertion 7, there exists an element $\tau$ of $G(V, f)$ such that $N(\tau)=\alpha, L \tau \subset L$ and $L_{i} / L_{i} \tau$ is isomorphic to $L_{i} / L_{i} \sigma_{i}$ as $\mathfrak{b}_{i}$-module for $1 \leqq i \leqq r$. By Proposition 2.13 and Proposition 3.11, there exists, for each $i$, an element $\varepsilon_{i}$ of $G^{0}\left(V_{i}, f\right)$ such that $L_{i} \varepsilon_{i}=L_{i}, L_{i} \tau \varepsilon_{i}$ $=L_{i} \sigma_{i}$. Then we have $L_{i} \sigma_{i} \varepsilon_{i}^{-1} \tau^{-1}=L_{i}$ and $N\left(\sigma_{i} \varepsilon_{i}^{-1} \tau^{-1}\right) \equiv 1 \bmod \mathfrak{p}_{\imath}^{\lambda i}$. By Assertion 5 , there exist elements $\rho$ and $\eta$ of $G^{0}(V, f)$ such that $L \rho=L, L \eta=L, L_{i}\left(\rho-\sigma_{i} \varepsilon_{i}^{-1} \tau^{-1}\right)$ $\subset \mathfrak{p}_{i}^{\lambda} L_{i}, L_{i}\left(\eta-\varepsilon_{i}\right) \subset \mathfrak{p}_{i}^{\lambda} L_{i}$. Put $\sigma=\rho \tau \eta$. We have then $N(\sigma)=\alpha, L \sigma \subset L$. If 
$z \in L_{i}$, we have

$$
z \sigma \equiv z \rho \tau \eta \equiv z \rho \tau \varepsilon_{i} \equiv z\left(\sigma_{i} \varepsilon_{i}^{-1} \tau^{-1}\right) \tau \varepsilon_{i} \equiv z \sigma_{i} \quad \bmod \mathfrak{p}_{i}^{i} L_{i},
$$

so that $L_{i}\left(\sigma-\sigma_{i}\right) \subset \mathfrak{p}_{i}^{\lambda_{i}} L_{i}$ for every $i$. This proves our assertion.

By Assertions 1, 2, 3 and 8, our Theorem 1 is completely proved.

We get a little weaker result than Theorem 1 for general maximal lattices, namely:

Theorem 2. Suppose that $A$ is indefinite. Let $M$ be a maximal lattice in $V$ not necessarily belonging to the principal genus. Let $\mathfrak{p}_{1}, \cdots, \mathfrak{p}_{r}$ be distinct prime $i d e a l s$ of $F$ and let $\alpha$ be an element of $\mathfrak{g}$. Let $\sigma_{i}$, for $1 \leqq i \leqq r$, be an element of $G\left(V_{\mathfrak{p}_{i}}, f\right)$. Suppose that $\alpha \equiv 1 \bmod \mathrm{t}, M_{\mathfrak{p}_{i}} \sigma_{i} \subset M_{\mathfrak{p}_{i}}, N\left(\sigma_{i}\right)=\alpha$ for $1 \leqq i \leqq r$. Then, for any set of positive integers $\left\{\lambda_{1}, \cdots, \lambda_{r}\right\}$, there exists an element $\sigma$ of $G(V, f)$ such that $M \sigma \subset M, N(\sigma)=\alpha, M_{\mathfrak{p}_{i}}\left(\sigma-\sigma_{i}\right) \subset \mathfrak{p}_{i}^{\lambda i} M_{\mathfrak{p}_{i}}$ for $1 \leqq i \leqq r$.

Proof. Let $\mathfrak{D}$ be the order of $M$. Take a member $L$ of $\mathfrak{L}_{0}(\mathfrak{D})$. Let $\left\{\mathfrak{p}_{r+1}\right.$, $\left.\cdots, \mathfrak{p}_{w}\right\}$ be the set of prime ideals $\mathfrak{p}$ of $F$ such that $M_{\mathfrak{p}} \neq L_{\mathfrak{p}}$ and $\mathfrak{p} \notin\left\{\mathfrak{p}_{1}, \cdots, \mathfrak{p}_{r}\right\}$. For each $\mathfrak{p}_{r+i}$, we can find, in view of Proposition 2.10 and Proposition 3.5, an element $\sigma_{r+i}$ of $G\left(V_{r+i}, f\right)$ such that $M_{r+i} \sigma_{r+i} \subset M_{r+i}, N\left(\sigma_{r+i}\right)=\alpha$. Take an integral ideal $\mathfrak{a}$ of $F$ such that $\mathfrak{a} L \subset M, \mathfrak{a} M \subset L, \mathfrak{a} L_{k} \sigma_{k} \subset L_{k}$ for $1 \leqq k \leqq w$. We may assume that the prime factors of $\mathfrak{a}$ belong to $\left\{\mathfrak{p}_{1}, \cdots, \mathfrak{p}_{r}, \mathfrak{p}_{r+1}, \cdots, \mathfrak{p}_{w}\right\}$. For a suitably large positive integer $h, \mathfrak{a}^{h}$ is a principal ideal $\mathfrak{g} \beta$; we have then $\beta L \subset M, \beta M \subset L, \beta L_{k} \sigma_{k} \subset L_{k}$ for $1 \leqq k \leqq w$. By Theorem 1, there exists an element $\tau$ of $G(V, f)$ such that $L \tau \subset L, N(\tau)=\beta^{2} \alpha, L_{k}\left(\tau-\beta \sigma_{k}\right) \subset \beta^{3} p_{k}^{k} L_{k}$ for $1 \leqq k \leqq w$. Put $\sigma=\beta^{-1} \tau$. Then $N(\sigma)=\alpha$ and $M_{k}\left(\sigma-\sigma_{k}\right)=\beta^{-1} M_{k}\left(\tau-\beta \sigma_{k}\right) \subset \beta^{-2} L_{k}\left(\tau-\beta \sigma_{k}\right)$ $\subset \beta \mathfrak{p}_{k}^{\lambda} L_{k} \subset \mathfrak{p}_{k}^{\lambda k} M_{k}$ for $1 \leqq k \leqq w$. As $M_{k} \sigma_{k} \subset M_{k}$, this implies $M_{k} \sigma \subset M_{k}$ for $1 \leqq k \leqq w$. If $\mathfrak{p} \notin\left\{\mathfrak{p}_{1}, \cdots, \mathfrak{p}_{w}\right\}$, we have $L_{\mathfrak{p}}=M_{\mathfrak{p}}$, and $\beta$ is a $\mathfrak{p}$-unit. We have therefore $M_{p} \sigma=L_{p} \beta^{-1} \sigma=L_{p} \tau \subset L_{p}=M_{p}$. Hence $M_{p} \sigma \subset M_{p}$ for any prime ideal $\mathfrak{p}$ of $F$. It follows that $M \sigma \subset M$. This completes the proof.

4.7. Class-number theorem. For every maximal lattice $L$ in $V$, put $N^{0}(L)$ $=N(L) \cap F$. Then $N^{0}(L)$ is a $\mathfrak{g}$-ideal. If $L$ is a member of $\mathfrak{L}\left(\mathfrak{D} ;\left\{e_{i}\right\}\right)$, we have $N(L)=N^{0}(L) \cdot \prod_{i=1}^{s} \mathfrak{Q}_{i}^{-e_{i}}$.

THEOREM 3. Suppose that $A$ is indefinite. Then, for every maximal order $\mathfrak{0}$ in $A$ and for every genus $\mathfrak{L}\left(\mathfrak{D} ;\left\{e_{i}\right\}\right)$ of maximal lattices in $V$, the mapping $L \rightarrow N^{0}(L)$ gives a one-to-one correspondence between the classes of maximal lattices in $\mathfrak{L}\left(\mathrm{D} ;\left\{e_{i}\right\}\right)$ and the ideal-classes modulo $\mathrm{t}$ in $\mathrm{F}$.

Therefore the number of classes in the genus $\mathfrak{L}\left(\mathbb{D} ;\left\{e_{i}\right\}\right)$ is equal to the number of ideal-classes modulo $\mathrm{t}$ in $F$.

Proof. Let $L$ and $M$ be members of $\mathfrak{L}\left(\mathfrak{0} ;\left\{e_{i}\right\}\right)$. If we have $L \rho=M$ for an element $\rho \in G(V, f)$ we have $N(L) N(\rho)=N(M)$, so that $N^{0}(L) N(\rho)=N^{0}(M)$. As $N(\rho) \equiv 1 \bmod \mathrm{t}$, the ideals $N^{0}(L)$ and $N^{0}(M)$ belong to the same ideal-class modulo $t$ in $F$. Conversely, suppose that $\alpha N^{0}(L)=N^{0}(M)$ for an element $\alpha \in F$ 
and $\alpha \equiv 1 \bmod$ t. Let $\beta$ be an element of $\mathfrak{g}$ such that $\beta M \subset L$. Let $\mathfrak{a}$ be an integral ideal of $F$ such that $\beta M \subset \mathfrak{a} L$. Let $\mathfrak{p}_{1}, \cdots, \mathfrak{p}_{r}$ be the prime factors of a. As we have $N(\beta M)=\beta^{2} \alpha N(L)$, we find, for each $\mathfrak{p}_{i}$, by Proposition 2.11 and Proposition 3.7, an element $\sigma_{i}$ of $G\left(V_{i}, f\right)$ such that $L_{i} \sigma_{i}=\beta M_{i}, N\left(\sigma_{i}\right)=\beta^{2} \alpha$. Since $\beta^{2} \alpha \equiv 1 \bmod t$, we can apply Theorem 2 to $\left\{L, \sigma_{i}, \beta^{2} \alpha\right\}$. Then we get an element $\sigma$ of $G(V, f)$ such that $L \sigma \subset L, N(\sigma)=\beta^{2} \alpha, L_{i}\left(\sigma-\sigma_{i}\right) \subset \mathfrak{p}_{i} \alpha L_{i}$. As $\mathfrak{p}_{i} a L_{i} \subset \mathfrak{p}_{i} \beta M_{i}=\mathfrak{p}_{i} L_{i} \sigma_{i}$, we have $L_{i} \sigma=L_{i} \sigma_{i}=\beta M_{i}$ by Lemma 1.2. If $\mathfrak{p}$ is a prime ideal which does not divide $\mathfrak{a}$, we have $L_{\mathfrak{p}}=\beta M_{\mathfrak{p}}$, because $L \supset \beta M \supset \mathfrak{a} L$. It follows that $N\left(L_{p}\right)=\beta^{2} \alpha N\left(L_{p}\right)$, and hence $\beta^{2} \alpha$ is a $p$-unit. As $L \sigma \subset L$ and $N(\sigma)=\beta^{2} \alpha$, we must have $L_{p} \sigma=L_{p}=\beta M_{p}$. Therefore we have $L_{p} \sigma=\beta M_{p}$ for any prime ideal $\mathfrak{p}$ of $F$, so that $L \sigma=\beta M$. Putting $\tau=\beta^{-1} \sigma$, we get $L \tau=M$. In view of Proposition 4.5, this proves our theorem.

4.8. Classes with respect to $\boldsymbol{G}^{0}(\boldsymbol{V}, \boldsymbol{f})$. If we take $G^{0}(V, f)$ instead of $G(V, f)$, we find that the class-number of each genus is equal to one. In fact, by Theorem 3 and its proof, we obtain easily

Proposition 4.10. Suppose that $A$ is indefinite. Let $L$ and $M$ be maximal lattices in $V$ with the same order. Then, there exists an element $\sigma$ of $G^{0}(V, f)$ such that $L \sigma=M$, if and only if $N(L)=N(M)$.

Notation being as in $\S 4.3$, let $\$^{\circ}$ be the subgroup of $\$$ consisting of elements $\left(\sigma_{\mathfrak{p}}, \sigma_{\kappa}\right)$ such that $\sigma_{\mathfrak{p}} \in G^{0}\left(V_{\mathfrak{p}}, f\right)$ for every $\mathfrak{p}$ and $\sigma_{\kappa} \in G^{0}\left(V_{\kappa}, f\right)$ for every $\kappa$. Then $\$^{0}$ can be regarded as the adele-group of $G^{0}(V, f)$. Put $G^{0}=G^{0}(V, f)$ and $\mathfrak{H}_{L}^{0}=\mathfrak{H}_{L} \cap \mathfrak{S}^{0}$. Then Proposition 4.10 implies the equality

$$
\mathbb{S}^{0}=\mathfrak{H}_{L}^{0} \cdot G^{0}
$$

for every maximal lattice $L$ in $V$.

4.9. Elementary divisors of lattices. Let $L$ and $M$ be members of the same genus $\mathfrak{L}\left(\mathfrak{D} ;\left\{e_{i}\right\}\right)$. For every prime ideal $\mathfrak{p}$ of $F$, we can define, as in $\S 2.5$ and $\S 3.2$, the set of elementary divisors $\left\{L_{p}: M_{p}\right\}$. We put $\{L: M\}_{p}=\left\{L_{p}: M_{p}\right\}$ and call it the $p$-part of the set of elementary divisors of $M$ relative to $L$. The (global) set of elementary divisors of $M$ relative to $L$ is defined as the join of $\{L: M\}_{\mathfrak{p}}$ for all prime ideals $\mathfrak{p}$ of $F$ and denoted by $\{L: M\}$.

THeOREM 4. Suppose that $A$ is indefinite. Let $L, M, K$ be maximal lattices in $V$ belonging to the same genus. Then, we have $\{L: M\}=\{L: K\}$ if and only if there exists an element $r$ of $G^{0}(V, f)$ such that $L r=L$ and $M r=K$.

Proof. The 'if' part is clear. Suppose that $\{L: M\}=\{L: K\}$. Let $\Psi$ be the set of prime ideals $\mathfrak{p}$ of $F$ for which $L_{p}=M_{\mathfrak{p}}=K_{\mathfrak{p}}$ does not hold. By Lemma 1.1, $\Psi$ is a finite set. By Proposition 2.13 and Proposition 3.11, there exists, for each $\mathfrak{p} \in \Psi$, an element $\gamma_{p}$ of $G^{0}\left(V_{p}, f\right)$ such that $L_{p} \gamma_{p}=L_{p}$ and 
$M_{p} \gamma_{p}=K_{p}$. Take a positive integer $c$ such that $\mathfrak{p}^{c} M_{\mathfrak{p}} \subset L_{\mathfrak{p}}$ and $\mathfrak{p}^{c} L_{\mathfrak{p}} \subset K_{\mathfrak{p}}$ for every $\mathfrak{p} \in \Psi$. By Theorem 2, there exists an element $\gamma$ of $G(V, f)$ such that $N(\gamma)=1, L \gamma \subset L, L_{p}\left(\gamma-\gamma_{p}\right) \subset \mathfrak{p}^{2 c+1} L_{p}$ for every $\mathfrak{p} \in \Psi$. Then obviously $L \gamma=L$, and $M_{\mathfrak{p}}\left(\gamma-\gamma_{\mathfrak{p}}\right) \subset \mathfrak{p}^{-c} L_{\mathfrak{p}}\left(\gamma-\gamma_{\mathfrak{p}}\right) \subset \mathfrak{p} \cdot \mathfrak{p}^{c} L_{\mathfrak{p}} \subset \mathfrak{p} K_{\mathfrak{p}}=\mathfrak{p} M_{\mathfrak{p}} \gamma_{\mathfrak{p}}$. By Lemma 1.2, we have $M_{p} \gamma=M_{p} \gamma_{p}=K_{\mathfrak{p}}$ for every $\mathfrak{p} \in \Psi$. If $\mathfrak{p} \notin \Psi$, we have $M_{\mathfrak{p} \gamma}=L_{p} \gamma=L_{p}=K_{\mathfrak{p}}$. Hence $M_{\mathfrak{p} r}=K_{\mathfrak{p}}$ holds for any prime ideal $\mathfrak{p}$ of $F$, so that $M_{r}=K$. This proves the 'only if' part.

Proposition 4.11. Suppose that $A$ is indefinite. Let $L$ and $M$ be maximal lattices in $V$ belonging to the same genus. Define the subgroups $\mathfrak{H}_{L}$ and $\mathfrak{U}_{M}$ of the adele-group $\$$ ss in $\S 4.3$. Put $\Gamma_{L}=\mathfrak{H}_{L} \cap G, \Gamma_{M}=\mathfrak{H}_{M} \cap G$. Then we have, for every $\xi \in \mathbb{B}$,

$$
\mathfrak{H}_{L} \xi \mathfrak{H}_{M}=\mathfrak{H}_{L} \xi \Gamma_{M}=\Gamma_{L} \xi \mathfrak{H}_{M}
$$

Proof. It is clear that $\mathfrak{H}_{L} \xi \mathfrak{H}_{M} \supset \mathfrak{H}_{L} \xi \Gamma_{M}$. Let $u$ be an element of $\mathfrak{H}_{M}$. As $M u=M$, we see easily, on account of the definition of $\mathfrak{H}_{M}$, that $\{M: L \xi u\}$ $=\{M: L \xi\}$. By Theorem 4, there exists an element $\gamma$ of $\Gamma_{M}$ such that $L \xi u=L \xi \gamma$. It follows that $\mathfrak{H}_{L} \xi u=\mathfrak{H}_{L} \xi \gamma \subset \mathfrak{H}_{L} \xi \Gamma_{M}$. This shows $\mathfrak{H}_{L} \xi \mathfrak{u}_{M} \subset \mathfrak{H}_{L} \xi \Gamma_{M}$, and hence $\mathfrak{H}_{L} \xi \mathfrak{H}_{M}=\mathfrak{H}_{L} \xi \Gamma_{M}$. Similarly we get $\mathfrak{U}_{M} \xi^{-1} \mathfrak{U}_{L}=\mathfrak{U}_{M} \xi^{-1} \Gamma_{L}$, so that $\mathfrak{H}_{L} \xi \mathfrak{H}_{M}=\Gamma_{L} \xi \mathfrak{H}_{M}$. This completes the proof.

The above theorem and proposition are generalization of [9, Proposition 1.4, Proposition 2.3]. These are necessary for our future investigation of the Hecke-ring of $G$.

\section{Osaka University}

\section{References}

[1] A. Borel, Some properties of adele groups attached to algebraic groups, Bull. Amer. Math. Soc., 67 (1961), 583-585.

[2] N. Bourbaki, Algèbre, Chap. 9, Formes sesquilinéaires et formes quadratiques, Hermann, Paris, 1959.

[3] M. Eichler, Quadratische Formen und orthogonale Gruppen, Berlin-GöttingenHeidelberg (Springer), 1952.

[4] M. Eichler, Die Ähnlichkeitsklassen indefiniter Gitter, Math. Z., 55 (1952), 216-252.

[5] M. Eichler, Allgemeine Kongruenzklasseneinteilungen der Ideale einfacher Algebren über algebraischen Zahlkörpern und ihre L-Reihen, J. Reine Angew. Math., 179 (1938), 227-251.

[6] M. Kneser, Klassenzahlen indefiniter quadratischer Formen in drei oder mehr Veränderlichen, Arch. Math., 7 (1956), 323-332.

[7] K. G. Ramanathan, Quadratic forms over involutorial division algebras, J. Indian Math. Soc., 20 (1956), 227-257.

[8] G. Shimura, On the zeta-functions of the algebraic curves uniformized by certain automorphic functions, J. Math. Soc. Japan, 13 (1961), 275-331.

[9] G. Shimura, On Dirichlet series and abelian varieties attached to automorphic 
forms, Ann. Math., 76 (1962), 237-294.

[10] T. Tsukamoto, On the local theory of quaternionic anti-hermitian forms, J. Math. Soc. Japan, 13 (1961), 387-400.

[11] A. Wei1, Discontinuous subgroups of classical groups, lecture note, Univ. of Chicago, 1958.

[12] A. Weil, Adeics and algebraic groups, lecture note, Institute for Advanced Study, Prirceton, 1961. 